\title{
Aufsätze
}

Markus Baumann, Marc Debus und Jochen Müller

\section{Das legislative Verhalten von Bundestagsabgeordneten zwischen persönlichen Charakteristika, Wahlkreisinteressen und Parteilinie}

Eine Untersuchung am Beispiel der Auseinandersetzung um die Präimplantationsdiagnostik

\section{Kurzfassung}

Die meisten Ansätze zur Erklärung legislativen Handelns und Entscheidens in parlamentarischen Demokratien konzentrieren sich auf institutionell basierte Anreize und die ideologisch-programmatischen Präferenzen der involvierten politischen Akteure. Im vorliegenden Beitrag argumentieren wir, dass insbesondere bei ethischreligiös unterlegten Thematiken, bei denen vielfach nur ein geringes Maß an Parteidisziplin herrscht, persönliche Eigenschaften und Erfahrungen eine ausschlaggebende Rolle bei der Gesetzesinitiierung, parlamentarischen Debatten und dem Abstimmungsverhalten spielen. Zudem gehen wir der Frage nach, ob sich das Handeln der Abgeordneten auch bei ethisch-religiösen Fragestellungen an den Einstellungen ihrer Wählerschaft orientiert. Wir testen unsere Erwartungen anhand eines Datensatzes, der den legislativen Prozess zur Regelung der Präimplantationsdiagnostik im Deutschen Bundestag nachzeichnet. Unsere Ergebnisse zeigen, dass sowohl Konfession, Geschlecht und Elternschaft der Abgeordneten als auch der Grad der Kirchengebundenheit ihrer lokalen Wählerschaft eine nicht unbedeutende Rolle für das legislative Verhalten der Bundestagsmitglieder in dieser Debatte spielte. 


\section{Inhalt}

1. Einleitung

2. Begrenzte Zulassung oder Verbot: Die Debatte zur Präimplantationsdiagnostik im deutschen Bundestag

3. Bestimmungsfaktoren des legislativen Handelns von Parlamentsabgeordneten

a) Nutzenmaximierendes responsives Handeln von Abgeordneten

b) Persönliche Charakteristika als Determinanten des Handelns von Abgeordneten

4. Daten und methodische Vorgehensweise

5. Ergebnisse

a) Determinanten der Initiierung der permissiven Gesetzesvorlage zur Regulierung der PID

b) Determinanten der in den Reden vertretenen Positionen zur PID

c) Determinanten der Haltung der Abgeordneten in der namentlichen Abstimmung

\section{Einleitung*}

Ein immer wieder in der Öffentlichkeit und in den Medien thematisiertes Phänomen des parlamentarischen Prozesses in der Bundesrepublik Deutschland betrifft den Widerspruch zwischen der im Grundgesetz niedergelegten Verpflichtung eines Abgeordneten seinem Gewissen gegenüber und der ausgeprägten Fraktionsdisziplin, die bei Abstimmungen in den Parlamenten auf Bundes- wie Landesebene zu beobachten ist (Sieberer 2010; Stecker 2011 a, 2011 b). So gibt es zahlreiche Fälle, in denen einzelne Mitglieder des Parlaments - etwa im Rahmen einer Presseerklärung, eines Interviews, einer Rede in einer Parlamentsdebatte oder einer schriftlichen Erklärung zur Abstimmung - ihren persönlichen Standpunkt deutlich machen, um

* Der Beitrag ist im Rahmen des am Mannheimer Zentrum für Europäische Sozialforschung angesiedelten und von der DFG geförderten Projekts 'Intra-party Heterogeneity and its Political Consequences in Europe' entstanden (DE 1667/2-1). Die Autoren bedanken sich bei der DFG für die Förderung des Projekts sowie bei den anonymen Gutachtern und der Redaktion der ZPol für hilfreiche Anregungen und Kritik. 
dann aber bei der Abstimmung der vorgegebenen Linie ihrer Fraktion, mit der sie nicht oder nicht vollständig übereinstimmen, zuzustimmen (Sieberer 2013). Ein prominentes Beispiel ist das Verhalten von acht Bundestagsabgeordneten der Fraktion Bündnis 90/Die Grünen im Rahmen der Abstimmung über den Antrag „Einsatz bewaffneter deutscher Streitkräfte bei der Unterstützung der gemeinsamen Reaktion auf terroristische Angriffe gegen die USA“ am 16. November 2001, die Bundeskanzler Schröder (SPD) mit der Vertrauensfrage verband. Wären diese acht Abgeordneten der Grünen ihren Präferenzen gefolgt, hätten sie gegen den Antrag stimmen müssen, was zum Sturz der Regierung geführt hätte. Daher entschlossen sich vier der acht grünen Fraktionsmitglieder, entgegen ihrem Standpunkt zu diesem Antrag für diesen zu stimmen, während die verbleibenden vier Abgeordneten bei ihrem Nein blieben, so dass der Bundesregierung unter Kanzler Schröder in Verbindung mit dem Antrag zum Einsatz der Bundeswehr in Afghanistan durch eine Mehrheit der Mitglieder des Deutschen Bundestages das Vertrauen ausgesprochen wurde.

Für ein solches Verhalten von Abgeordneten gibt es eine Vielzahl von Ursachen und Erklärungen, die in der politikwissenschaftlichen Analyse parlamentarischen Verhaltens bereits breit analysiert und diskutiert wurden. Hierzu zählen etwa - wie im oben genannten Beispiel - die Gefährdung der Regierung durch ein von der Parteilinie abweichendes Verhalten bei parlamentarischen Abstimmungen, parteiinterner Druck auf einzelne Abgeordnete dahingehend, dass bei einem Abweichen von der Parteiposition in kommenden Wahlkämpfen mit keiner oder geringerer Unterstützung zu rechnen ist, sowie Unterschiede in den politischen Zielen der Partei, der ein Abgeordneter angehört, und den Präferenzen der Wähler im Wahlkreis des Abgeordneten (Saalfeld 1995; Manow 2007, 2012; Patzelt 2007; Depauw/Martin 2009; Sieberer 2010; Andeweg/Thomassen 2011; Proksch/Slapin 2012). Ausgenommen von solchen - direkt oder indirekt - angewendeten disziplinierenden Maßnahmen sind hingegen vielfach Themen, die religiös sowie ethisch-moralisch motivierte Werte und Einstellungen tangieren und die von den sozialen Konfliktlinien, die Wahlverhalten, Parteienwettbewerb und legislative Entscheidungsfindung eines Landes maßgeblich strukturieren (Lipset/Rokkan 1967; Pappi/Shikano 2002), nicht vollständig abgedeckt werden. Beispiele hierfür sind insbesondere Gesetzesinitiativen zu ethischen Fragen, die die Regulierung der Anwendung neuer Techniken in der Medizin betreffen und bei denen neben wirtschafts- und gesellschaftspolitischen Aspekten auch religiöse sowie ethisch-moralische Sichtweisen von Relevanz sind, so dass die Abstimmungen zu diesen Fragen vielfach als „Gewissensentscheidungen“ deklariert werden, bei denen kein „Fraktionszwang“" besteht (Meidert/Nebel 2013; Preidel 2011, 2012). 
Wir betrachten daher in unserem Beitrag eine Thematik, die religiöse und ethischmoralische Werte und Einstellungen betrifft, um die Fragestellung zu beantworten, inwiefern das legislative Verhalten von Bundestagsabgeordneten von ihren Biografien, persönlichen Eigenschaften und Erfahrungen sowie der Präferenzlage in ihrem Wahlkreis abhängt. Konkret untersuchen wir den Einfluss dieser Faktoren auf die Gesetzesinitiativtätigkeit, die Inhalte von Parlamentsreden und das letztendliche Abstimmungsverhalten der Abgeordneten bei der Auseinandersetzung um die Regelung der Präimplantationsdiagnostik (PID) im Frühsommer 2011. Das Hauptargument unseres Beitrages steht damit im engen Bezug zur Forschung zur Zusammensetzung und deskriptiven Repräsentativität (Rebenstorf/Weßels 1989) des Bundestages sowie des Rollenverständnisses seiner Mitglieder (Patzelt 1989) und überprüft die Auswirkungen unterschiedlicher Merkmale von Bundestagsabgeordneten auf ihr legislatives Verhalten. Im Unterschied zu früheren Beiträgen ist das primäre Ziel hier allerdings keine vollständige Beschreibung der betreffenden Eigenschaften, sondern ein Herausarbeiten derjenigen Merkmale, die in dem hier untersuchten Gesetzgebungsprozess relevante Einflüsse darstellen. Mit der Herstellung einer kausalen Beziehung zwischen persönlichen Merkmalen von Abgeordneten, die in nahezu identischer Form zu den gängigsten erklärenden Variablen in der Wahlforschung gehören (Falter/Schoen 2005; Pappi/Shikano 2007), und dem legislativen Verhalten der Abgeordneten demonstriert unser Beitrag zugleich die Relevanz einer abgeordnetensoziologischen Betrachtung des Bundestages. Die Ergebnisse zeigen, dass das Handeln und Entscheiden von Abgeordneten im Rahmen der Debatte um die Regulierung der Präimplantationsdiagnostik in Deutschland unter Kontrolle parteipolitischer Faktoren - von der Struktur der Wahlkreise in Form der Kirchengebundenheit der jeweiligen lokalen Wählerschaft sowie persönlichen Eigenschaften wie der Konfession, dem Geschlecht und der Elternschaft der Abgeordneten beeinflusst wurde.

Um diese Beziehung aufzuzeigen, gehen wir wie folgt vor: Zunächst geben wir einen kurzen Überblick zur Debatte um die PID in Deutschland und diskutieren im Anschluss daran den Stand der Forschung zu den Determinanten der legislativen Aktivität von Parlamentsabgeordneten mit Rückgriff auf die Konzeption des responsiven Handelns in modernen Demokratien, den Prinzipal-Agenten-Ansatz und die persönlichen Eigenschaften und Erfahrungen von Abgeordneten. Die daraus abgeleiteten Erwartungen hinsichtlich der legislativen Initiativtätigkeit, der in Reden eingenommenen Positionen sowie den in den namentlichen Abstimmungen getroffenen Entscheidungen werden in Abschnitt fünf einer empirischen Analyse unterzogen. Zuvor wird im vierten Abschnitt näher auf die verwendeten Daten und Methoden eingegangen. Die Schlussbetrachtung fasst die gewonnenen Erkenntnisse 
zusammen und diskutiert Anreize für weitere Studien zur Analyse parlamentarischer Debatten und der Rückkopplung ihrer Inhalte an die persönlichen Merkmale von Parlamentsabgeordneten.

\section{Begrenzte Zulassung oder Verbot: Die Debatte zur Präimplantationsdia- gnostik im deutschen Bundestag}

Die aus medizinisch-technischer Perspektive seit einigen Jahren mögliche Präimplantationsdiagnostik wurde in Deutschland bis Mitte 2010 durch die Vorschriften des Embryonenschutzgesetzes geregelt, woraus aufgrund des Verbots der Verwendung menschlicher Embryonen eine Nicht-Zulassung der PID abgeleitet wurde. In Deutschland wurde zunächst keine explizite Regulierung der Reproduktionsmedizin angestrebt. Durch die Entscheidung des Bundesgerichtshofs vom 6. Juli 2010 mit dem Ergebnis, dass eine Anwendung der Präimplantationsdiagnostik im Rahmen der Herbeiführung einer Schwangerschaft und zum Ausschluss von drohenden Erbkrankheiten nicht gegen die Strafnormen des Embryonenschutzgesetzes verstößt, ${ }^{1}$ wurde die PID unter diesen Bedingungen straffrei.

Im parlamentarischen Prozess resultierte die mit der faktischen Zulassung der PID verbundene öffentliche Diskussion in drei Gesetzesvorlagen. Von einer Gruppe Abgeordneter um Ulrike Flach, der gesundheitspolitischen Sprecherin der FDP, wurde eine - gegenüber der PID - liberale oder permissive Initiative eingebracht. Die restriktivste der drei Initiativen geht auf die grüne Abgeordnete Katrin GöringEckardt zurück. Einen Mittelweg zwischen diesen beiden Vorlagen stellte die von René Röspel (SPD) eingebrachte Vorlage dar. Die liberal-permissive Gesetzesinitiative strebte ebenso wie - wenn auch in etwas abgeschwächter Form - die Initiative des Abgeordneten Röspel eine eingeschränkte Zulassung der PID an, während die konservativ-restriktive Vorlage ein vollständiges Verbot vorsah. Alle drei Initiativen wurden in erster und zweiter Lesung im April bzw. Juli 2011 im Bundestag beraten. Die auf die zweite Lesung folgende erste namentliche Abstimmung fand zwischen allen drei Vorlagen statt. Da in der ersten Abstimmung keine der drei Gesetzesinitiativen die absolute Mehrheit der abgegeben Stimmen erreichte, wurde in dritter Lesung über die Annahme des permissiven Vorschlags abgestimmt.

Wie aus Tabelle 1 entnommen werden kann, folgte bereits die Unterzeichnung der drei Vorlagen durch die Abgeordneten weder einer Parteilinie noch der Trennlinie zwischen Opposition und Regierung. Es wird deutlich, dass Mitglieder der CDU/CSU-Fraktion mehrheitlich den konservativen Vorschlag unterstützten und

1 Entscheidung des Bundesgerichtshofs vom 6. Juli 2010 (AZ 5 StR 386/09). 
Angehörige der sozialdemokratischen sowie insbesondere der liberalen Fraktionen eher zu den permissiven Vorschlägen neigten, was mit der jeweiligen gesellschaftspolitischen Grundausrichtung der Partei übereinstimmt (Pappi/Shikano 2002, 2004; Bräuninger/Debus 2012: 35-57). Allerdings sind die Anteile der Abgeordneten innerhalb dieser drei Fraktionen, die sich für einen inhaltlich permissiveren oder restriktiveren Vorschlag ausgesprochen haben, überraschend hoch. Dass zudem eine relative Mehrheit der Abgeordneten der Fraktion von Bündnis 90/Die Grünen, die als Partei in ihren Programmen auf allen Ebenen des politischen Systems Deutschlands gesellschaftspolitisch progressiv und liberal ausgerichtet ist (Bräuninger/Debus 2012: $54 \mathrm{ff}$.), die restriktive Gesetzesinitiative stützte, macht deutlich, von welch eher geringer Bedeutung die Partei- und Fraktionszugehörigkeit bei der schlussendlichen Entscheidung über die Regulierung der PID war. Zugleich ergibt sich daraus die Frage nach den Faktoren, die das legislative Verhalten der Abgeordneten determinierten. Aus diesem Grund diskutieren wir im folgenden Abschnitt die Ergebnisse von Studien zu den Determinanten des legislativen Handels und Entscheidens von Parlamentsabgeordneten. Auf deren Basis leiten wir Erwartungen ab, die unsere empirische Analyse der Debatte und Entscheidungsfindung zur Präimplantationsdiagnostik im Bundestag strukturieren.

Tabelle 1: Parteizugehörigkeit der Initiatoren und Unterzeichner der drei Gesetzesvorlagen zur Regulierung der PID

\begin{tabular}{lrrrrrrr}
\hline $\begin{array}{l}\text { Inhaltliche Ausrich- } \\
\text { tung der Gesetzesvor- } \\
\text { lage }\end{array}$ & $\begin{array}{c}\text { CDU/ } \\
\text { CSU }\end{array}$ & $\begin{array}{c}\text { Bündnis } \\
\text { 90/Die } \\
\text { Grünen }\end{array}$ & $\begin{array}{c}\text { Die Lin- } \\
\text { ke }\end{array}$ & SPD & FDP & Insgesamt & $\begin{array}{c}\text { Anzahl unter- } \\
\text { stützende MdBs }\end{array}$ \\
\hline Konservativ & $53,4 \%$ & $41,2 \%$ & $23,7 \%$ & $13,0 \%$ & $4,3 \%$ & $31,6 \%$ & 196 \\
Moderat & $2,5 \%$ & $14,7 \%$ & $1,3 \%$ & $11,6 \%$ & $1,1 \%$ & $5,6 \%$ & 35 \\
Liberal-permissiv & $15,1 \%$ & $26,5 \%$ & $35,5 \%$ & $43,8 \%$ & $77,4 \%$ & $34,9 \%$ & 217 \\
$\begin{array}{l}\text { An keiner Initiative } \\
\text { beteiligt }\end{array}$ & $29,0 \%$ & $17,7 \%$ & $39,5 \%$ & $31,5 \%$ & $17,2 \%$ & $27,9 \%$ & 173 \\
\hline
\end{tabular}

Anmerkung: Die Werte geben jeweils den Anteil der Mitglieder der Fraktion an, die die jeweilige Gesetzesvorlage unterstützen.

Quelle: Gesetzentwürfe zur Präimplantationsdiagnostik (BT-Drucksachen 17/5450, 17/5451 und 17/5452). 
Das legislative Verhalten von Bundestagsabgeordneten

\section{Bestimmungsfaktoren des legislativen Handelns von Parlamentsabgeord- neten}

Zur Herleitung unserer Erwartungen bauen wir auf einer Reihe von theoretischen Ansätzen auf, die sich einerseits auf nutzenmaximierende Strategien und das responsive Handeln politischer Akteure sowie andererseits auf die Bedeutung persönlicher Merkmale und Biografien bei der Herausbildung von Präferenzen beziehen. In den folgenden Unterabschnitten werden diese Ansätze vor dem Hintergrund der Bestimmungsfaktoren legislativen Handelns von Parlamentsabgeordneten näher beleuchtet.

\section{a) Nutzenmaximierendes responsives Handeln von Abgeordneten}

Ausgangspunkt unserer Überlegungen bildet die Annahme, dass das Handeln politischer Akteure von der Maximierung ihres Nutzens geleitet ist. Letzterer ergibt sich aus den erzielten Wählerstimmen, der Anzahl politischer Ämter und der schließlich durchgesetzten und implementierten Politikinhalte (Strøm/Müller 1999). Parteien sowie ihre Kandidaten und gewählten parlamentarischen Vertreter sollten demnach bemüht sein, sich so zu verhalten, dass der Stimmenanteil und somit die Chance auf (Wieder-)Wahl und (erneute) Regierungsbeteiligung maximiert wird. Unter der Annahme, dass das individuelle Wahlverhalten bei Bundestagswahlen auch von den in der sozialen Lage der Wähler verwurzelten Interessen und den inhaltlich-programmatischen Vorstellungen der Wählerschaft beeinflusst wird (Thurner 2000; Weßels 2000; Pappi/Brandenburg 2010; Debus 2012), sollten die Wahlkämpfe von Bundestagskandidaten und insbesondere der Wahlkreisbewerber auf die spezifischen Präferenzen und Interessenlagen der lokalen Wählerschaft ausgerichtet sein, die zu einem gewissen Grad von der Parteilinie abweichen können. Ähnliche Erwartungen ergeben sich unter Rückgriff auf normativ ausgerichtete Idealtypen des responsiven Regierens in modernen Demokratien, die von einer optimalen Delegationskette der Interessen des Median-Wählers über die gewählten Abgeordneten hin zur Wahl der Regierung und der Produktion von Politikinhalten ausgehen (Bergman/Müller/Strøm 2000; Müller 2000; Powell 2004). So können etwa Gschwend und Zittel (2007) anhand einer Befragung von Bundestagskandidaten empirisch nachweisen, dass Direktkandidaten vor allem dann einen eigenständigen Wahlkampf führen, der von den Vorgaben der sie nominierenden Partei abweicht, je größer die Aussicht auf einen Gewinn des Wahlkreismandats ist. Neuere Studien zum Abstimmungsverhalten von Parlamentsabgeordneten zeigen zudem, dass trotz hoher Parteidisziplin im Bundestag und in anderen europäischen Parlamenten aufgrund möglicher Konsequenzen beim Abweichen von der Parteilinie lokale bzw. 
wahlkreisspezifische Besonderheiten in die Entscheidungsfindung eines Abgeordneten bei legislativen Vorlagen eingehen. So hebt Sieberer (2010) den Unterschied zwischen Wahlkreis- und Listenmandat bei Deutschen Bundestagswahlen hervor und kann nachweisen, dass direkt in einem Wahlkreis gewählte Abgeordnete sich mit signifikant höherer Wahrscheinlichkeit der - im Deutschen Bundestag sehr starken (Sieberer 2006) - Fraktionsdisziplin bei namentlichen Abstimmungen eher entgegenstellen als über die Landeslisten der Parteien gewählte Abgeordnete (Saalfeld 1995, 2003).

Neben ihrem Abstimmungsverhalten können Abgeordnete auch die legislative Initiativtätigkeit nutzen, um der lokalen bzw. regionalen Wählerschaft zu signalisieren, dass sie bemüht sind, deren mehrheitliche Präferenzen in den legislativen Prozess einzubringen. So kann Bowler (2010) am Beispiel der gesetzgeberischen Tätigkeit von Mitgliedern des britischen Unterhauses und der Reaktionen der Wählerschaft zeigen, dass ein hohes Maß an legislativer Aktivität vom jeweiligen Elektorat gewürdigt wird: Wähler schätzen ihre Abgeordneten umso mehr, je häufiger diese an legislativen Initiativen beteiligt waren. Bräuninger, Brunner und Däubler (2012) können auf der Basis einer Untersuchung der legislativen Initiativtätigkeit von Abgeordneten im belgischen Parlament zeigen, dass die Einführung von Vorzugsstimmen im Rahmen von Verhältniswahlsystemen Anreize dafür bietet, dass Abgeordnete sich in verstärktem Maße an der Initiierung von Gesetzesinitiativen beteiligen, um in der Wählerschaft auf Anerkennung zu stoßen und somit die Chancen zu erhöhen, aufgrund einer höheren Zahl von Vorzugsstimmen bei der kommenden Parlamentswahl wiedergewählt zu werden.

Zusammengefasst gibt es für verschiedene europäische Parlamente - trotz eines hohen Maßes an Fraktionsdisziplin - Evidenz dafür, dass Abgeordnete bemüht sind, die Präferenzen ihrer lokalen Wählerschaft in ihrem Handeln im Parlament zu berücksichtigen und dabei zuweilen ein Abweichen von der Parteilinie in Kauf zu nehmen. Offenbar haben Abgeordnete selbst in Parlamenten mit hoher Fraktionsdisziplin genügend Spielraum, der es ihnen erlaubt, zwei Prinzipalen - im vorliegenden Fall ihrer Partei bzw. Parlamentsfraktion sowie den Wählern in ihrem Wahlkreis - gerecht zu werden, obwohl diese mitunter verschiedene Interessen aufweisen können (Bergman/Müller/Strøm 2000; Carey 2009; auch Müller 2000). Da der Prinzipal Partei die Parlamentsabgeordneten von ihrer Rolle als Agent bei Abstimmungen, die als „Gewissensentscheidungen“ deklariert werden, de facto „entbindet“, sollten Abgeordnete den Präferenzen und Interessen der lokalen bzw. regionalen Wählerschaft den Vorrang gegenüber Parteiinteressen einräumen. Dies ermöglicht den Abgeordneten die Berücksichtigung der Werte und Einstellungen ihrer lokalen Wählerschaft im Rahmen ihrer Entscheidungsfindung, was sie dem Ziel der 
erneuten Nominierung und Wiederwahl näherbringen dürfte. Wir erwarten daher, dass die Bundestagsabgeordneten in der Auseinandersetzung um die Präimplantationsdiagnostik im Sinne der Maximierung ihrer Wiederwahlchancen in hohem Maße bemüht sind, die (vermuteten) Präferenzen der Wähler in ihrem Wahlkreis zu diesem Themenbereich abzubilden. Konkret formulieren wir die folgenden Erwartungen:

H1: Je stärker kirchengebunden (und damit skeptischer gegenüber der Anwendung der Präimplantationsdiagnostik) die Wählerschaft in einem Wahlkreis ist, desto eher werden den Wahlkreis vertretende Bundestagsabgeordnete

a) die restriktive, den Vorstellungen der römisch-katholischen und evangelischen Kirchen nahekommende Gesetzesinitiative unterzeichnen;

b) in Parlamentsreden skeptische Positionen gegenüber der Anwendung der Präimplantationsdiagnostik einnehmen;

c) die permissiv-liberale Gesetzesvorlage in der namentlichen Abstimmung ablehnen.

\section{b) Persönliche Charakteristika als Determinanten des Handelns von Abge- ordneten}

Der Fokus auf Parteien und deren nutzenorientiertes Handeln, der bei der Erforschung etablierter Demokratien mit starken Parteien bei vielen politikwissenschaftlichen Fragen angebracht ist, bringt die Vernachlässigung der Perspektive mit sich, dass politische Akteure auch immer Menschen oder Gruppen von Menschen sind, die aufgrund ihrer Erfahrungen, Biografien und Lebenssituationen bestimmte Präferenzen hinsichtlich staatlichen Handelns haben. Da diese Einstellungen insbesondere im Kontext ethisch-moralischer Fragestellungen das Handeln von Abgeordneten prägen sollten, berücksichtigen wir die entsprechenden Überlegungen bei unserer Untersuchung. Dabei wird im Vergleich zu den Ansätzen, die im vorigen Abschnitt diskutiert wurden, kein grundlegend anderes Entscheidungskalkül unterstellt. Vielmehr wird die Betrachtung auf die von persönlichen Charakteristika abhängige Präferenzbildung des Abgeordneten ausgedehnt. Burden (2007; auch Rocca/Sanchez 2008) zeigt an einer Reihe von Fallbeispielen, wie persönliche Eigenschaften und Erfahrungen das legislative Verhalten von Mitgliedern des amerikanischen Repräsentantenhauses und Senats sowohl bei der Initiierung von Gesetzesvorhaben als auch bei der Abstimmung über Gesetzesvorlagen beeinflussen können. So verdeutlicht Burden, dass Kongressmitglieder, die Raucher sind, dazu neigen, sich gegen eine stärkere Regulierung des Tabakkonsums auszusprechen und dementsprechende Gesetzesvorhaben eher nicht unterstützen (Burden 2007: 54 ff.). Zu- 
dem kann er nachweisen, dass die Politikfelder, mit denen sich Abgeordnete in den beiden Kammern des Kongresses beschäftigen, mit den persönlichen Hintergründen der Mitglieder von Senat und Repräsentantenhaus korrespondieren: So beschäftigen sich Abgeordnete, die Kinder im Schulalter haben, eher mit Fragen der Bildungsund Schulpolitik als andere Kongressabgeordnete (ebd.: $88 \mathrm{ff}$.).

In Übereinstimmung mit der Argumentation aus den „Standardmodellen“ des responsiven Handelns von politischen Akteuren und der Delegation von Politikzielen und Interessen, auf die wir im vorherigen Abschnitt bereits kurz eingegangen sind, kommt Burden zu dem Schluss, dass sich Abgeordnete des amerikanischen Kongresses gemäß den Präferenzen der Wählerschaft in ihrem Wahlbezirk oder Bundesstaat verhalten, so dass bei der nächsten Wahl die Chancen auf erneute Nominierung und Wiederwahl maximiert werden (2007: 42, 136). Dieses Ergebnis ist allerdings auch durch die aus dem Wahlsystem resultierende starke Kopplung zwischen Repräsentant und Elektorat zu erklären und besteht nicht zwingend in allen Politikfeldern (ebd.: $46 \mathrm{ff}$.).

Aus theoretischer Perspektive geht Burden (2007: 42 ff.) in Übereinstimmung mit Careys (2009) Ansatz von mehreren Prinzipalen aus, deren Interessen ein Abgeordneter als Agent bedienen muss. Parlamentsmitglieder befinden sich demnach in einem ,field of forces“(Kingdon 1989), wenn es darum geht, sich im Rahmen des parlamentarischen Handelns zu einer Gesetzesinitiative zu positionieren. Die auf die Entscheidungsfindung eines Parlamentariers einwirkenden Kräfte lassen sich in interne und externe Einflüsse unterscheiden. Die Präferenzen eines Abgeordneten werden demnach zunächst durch die internen Faktoren - die persönlichen Erfahrungen, Wertvorstellungen, „Ideologie“ und Eigeninteressen - geprägt. Unter Einfluss der externen Faktoren des Kräftefeldes, wie beispielsweise der vorgegebenen Linie einer Partei zu einer bestimmten Sachfrage oder der Wünsche von Interessengruppen, evaluieren Abgeordnete ihre parlamentarischen Handlungsoptionen und deren Konsequenzen. Je nach Stärke und Richtung der Wirkung externer Einflussfaktoren entspricht das Handeln des Abgeordneten seinen Präferenzen mehr (geringe oder gleichgerichtete externe Einflüsse) oder weniger (starke und gegensätzliche externe Einflüsse). Gemäß diesem Prozess der Präferenzbildung und Präferenzwirkung geht Burden davon aus, dass persönliche Charakteristika eines Abgeordneten immer dann von besonderer Bedeutung sind, wenn externe Einflüsse nicht oder nur in geringem Umfang vorhanden sind (siehe auch Abbildung 1).

Persönliche Charakteristika sollten vor allem dann von Bedeutung für das parlamentarische Handeln eines Abgeordneten sein, wenn eine Vorlage einem Politikfeld entstammt, das sich nicht oder nur schlecht in die gängigen, Wahlverhalten, Parteienwettbewerb und die Auseinandersetzung in Parlamenten strukturierenden, Kon- 
Abbildung 1: Einflussfaktoren auf die parlamentarische Aktivität von Abgeordneten nach Burden (2007: 43)



fliktlinien einordnen lässt. Dies schließt laut Burden (2007) unter anderem solche Themengebiete ein, die einen ethisch-moralischen Hintergrund haben. Bei legislativen Vorhaben zu solchen Themenbereichen sollten - um im Bild der PrinzipalAgenten-Theorie zu bleiben - neben Partei und Wählerschaft ein „dritter Prinzipal“, dem sich ein Abgeordneter verpflichtet fühlt, hinzukommen und eine entscheidende Rolle einnehmen. Dieser „dritte Prinzipal“" stellt die Summe der persönlichen Erfahrungen, Werte und Einstellungen eines Abgeordneten dar und kann - vor dem Hintergrund von Artikel 38, Absatz 1, Satz 2 des Grundgesetzes der Bundesrepublik Deutschland, der das „freie Mandat“ garantiert und den Fraktionszwang de jure unterbindet - als das Gewissen eines Abgeordneten bezeichnet werden (vgl. hierzu Ismayr 2000: 50 ff.). Laut Burden (2007) sind hier die religiöse Ausrichtung eines Parlamentariers, sein beruflicher und schulischer Werdegang sowie sein privates Umfeld, aus welchem er Erfahrungen schöpfen und für das er die positiven oder negativen Konsequenzen einer Gesetzesvorlage ermessen kann, von zentraler Bedeutung.

Da in parlamentarischen Regierungssystemen eine höhere Fraktionsdisziplin als in präsidentiellen Regierungssystemen herrscht (Sieberer 2006; Kam 2009), sollten Abgeordnete des Deutschen Bundestages in ihren legislativen Entscheidungen grundsätzlich zu einem geringen Ausmaß von persönlichen Einstellungen und Erfahrungen, sondern vielmehr durch parteipolitische Anreize und wahltaktische Überlegungen beeinflusst werden. Eine Ausnahme sollten Thematiken mit einem explizit religiösen oder ethisch-moralischen Hintergrund darstellen, bei denen im 
Deutschen Bundestag - ähnlich wie in anderen parlamentarischen Demokratien bei der Ausarbeitung von und der Abstimmung über Gesetzesinitiativen nicht nur eine fraktions-, sondern auch eine regierungs- und oppositionslagerübergreifende Zusammenarbeit festgestellt werden kann (Preidel 2012; siehe auch Bräuninger/ Debus 2010; Engeli/Green-Pedersen/Larsen 2012; Brunner 2013). Wir erwarten im Rahmen unserer Analyse der legislativen Initiativtätigkeit, parlamentarischen Debatte und Abstimmung zu den verschiedenen Vorschlägen der Regulierung der Präimplantationsdiagnostik, dass religiöse Einstellungen und hier insbesondere die $\mathrm{Zu}$ gehörigkeit zur römisch-katholischen Kirche als der Institution, die der Präimplantationsdiagnostik besonders skeptisch gegenübersteht, zusätzlich zu den üblichen Determinanten des legislativen Verhaltens, wie Fraktionsdisziplin und Rückkopplung an die Interessen der Wählerschaft, eine ausschlaggebende Rolle gespielt haben.

Zusätzlich gehen wir davon aus, dass das Geschlecht und der familiäre Hintergrund der Abgeordneten einen Einfluss auf deren legislatives Verhalten in dieser Sachfrage haben sollten. Theorien, die die Effekte sozialer Rollen und sozialer Identität für das individuelle Handeln hervorheben (vgl. etwa Mead 1934; Searing 1991; für eine Übersicht vgl. Esser 2001: 335 ff.), argumentieren, dass die Handlungsweisen von Individuen nicht nur von externen Bedingungen beeinflusst, sondern auch von internen Einstellungen determiniert werden, die wiederum in entscheidender Form vom sozialen Umfeld und auch von den sozialstrukturellen Merkmalen der jeweiligen Personen abhängen (vgl. auch Esser 1999: 54 ff.). Während die politische Psychologie im Rahmen des „Big Five“-Ansatzes vor allem auf die sich in der Kindheit herausbildenden oder auch vererbten Persönlichkeitseigenschaften abhebt (vgl. für eine Übersicht Schoen 2012), wird von Simon $(1985,1995)$ im Rahmen des „bounded rationality“-Konzepts hervorgehoben, dass sich - in Kombination mit der jeweils auf der Agenda stehenden Thematik - die Präferenzen und Handlungsmaximen eines Individuums aus der sozialen Gruppenzugehörigkeit sowie den biografischen und histografischen Erfahrungen ergeben können (vgl. Hartmann 2007: 20 f.).

Aufbauend darauf argumentieren wir, dass insbesondere weibliche Abgeordnete im Rahmen der Debatte um die Präimplantationsdiagnostik, deren Einführung insbesondere gesundheitliche Risiken für schwangere Frauen mindert, da mitunter mit Komplikationen einhergehende invasive Untersuchungen nicht mehr notwendig sind und das Risiko von Fehlgeburten gemindert wird, aufgrund der durch das Geschlecht vermittelten, direkten Betroffenheit als Vertreter der Interessen von Frauen parlamentarisch agieren sollten. Studien zur substanziellen Repräsentation - insbesondere von Frauen - zeigen, dass die Parteizugehörigkeit an Bedeutung verliert, 
wenn eine Gesetzesinitiative die soziale Gruppe begünstigt, zu der ein Parlamentsmitglied ein besonderes Verhältnis hat (vgl. Esaiasson/Heidar 2000; Dodson 2006; Wängnerud 2009). Insofern besteht - auf Basis des durch die soziale Identität induzierten Repräsentationsziels der Interessen dieser sozialen Gruppe - der Anreiz, dass insbesondere weibliche Bundestagsabgeordnete für eine liberal-permissive Regulierung der Präimplantationsdiagnostik eintreten sollten. Dies sollte-aufgrund der persönlichen Erfahrungen, die in das legislative Handeln und Entscheiden einbezogen werden (Simon 1985, 1995) - vor allem dann geschehen, wenn die weiblichen Bundestagsabgeordneten selbst Kinder haben und sich daher bereits mit möglichen Komplikationen im Laufe einer Schwangerschaft befassen mussten. Wir postulieren daher die folgenden Hypothesen:

$\mathrm{H} 2$ : Abgeordnete, die römisch-katholischer oder evangelischer Konfession sind und somit den beiden Kirchen angehören, die die PID strikt bis weitgehend ablehnen, sollten mit höherer Wahrscheinlichkeit

a) die permissive Gesetzesinitiative, die eine offenere Regulierung der PID vorsieht, nicht unterzeichnen;

b) skeptische Positionen gegenüber der Anwendung der Präimplantationsdiagnostik in ihren Parlamentsreden einnehmen;

c) die permissive Gesetzesvorlage ablehnen.

H3: Abgeordnete, die Kinder haben, sollten aufgrund ihrer Erfahrungen mit der Geburt und der Auseinandersetzung mit damit verbundenen möglichen Komplikationen mit höherer Wahrscheinlichkeit

a) die permissive Gesetzesinitiative, die eine Anwendung der PID in bestimmten Fällen ermöglicht, unterzeichnen;

b) befürwortende Haltungen gegenüber der Anwendung der Präimplantationsdiagnostik in ihren Parlamentsreden einnehmen;

c) der permissiven Gesetzesvorlage in der namentlichen Abstimmung zustimmen.

H4: Die in Hypothese 3 genannten Zusammenhänge sollten sich verstärken, wenn es sich um weibliche Abgeordnete handelt.

Um die aufgestellten Erwartungen zu testen, bedarf es einer Reihe von Informationen zu den parteipolitischen und persönlichen Eigenschaften der Abgeordneten, den Eigenschaften ihrer Wahlkreise sowie ihrer Positionierung und Entscheidung in der Bundestagsdebatte um die Regulierung der Präimplantationsdiagnostik. Im folgen- 
den Abschnitt gehen wir daher näher auf die verwendeten Daten sowie die methodischen Techniken ein, die zur Überprüfung der Hypothesen benötigt werden.

\section{Daten und methodische Vorgehensweise}

Im Rahmen unserer Untersuchung betrachten wir drei Stufen des legislativen Prozesses: die Initiierung von Gesetzesinitiativen, die Redebeiträge in den Debatten und das Abstimmungsverhalten. Entsprechend müssen Informationen dahingehend eingeholt werden, welche Abgeordneten eine der drei Gesetzesinitiativen zur Regulierung der Präimplantationsdiagnostik in Deutschland (mit-)eingebracht haben, welche Haltung zur PID sie in Reden zum Ausdruck bringen und für welche Initiative sie schlussendlich votiert haben. Während die Initiatoren einer Gesetzesinitiative und das Abstimmungsverhalten sehr leicht über das Informationsangebot und die Protokolle des Deutschen Bundestages zu ermitteln sind, ist es deutlich schwieriger, die eigentlichen Positionen der Abgeordneten zur PID zu erheben. Letzteres ist jedoch notwendig, um zu überprüfen, ob die Abgeordneten über die drei Stufen des legislativen Prozesses hinweg - also bei Initiierung, Debatte und Abstimmung - konsistent dieselbe Position eingenommen haben oder ob es Unterschiede zwischen den ersten beiden Stufen auf der einen Seite und der dritten und entscheidenden Phase der Abstimmung auf der anderen Seite gab. Wenn Letzteres der Fall ist, dann würde dies darauf hindeuten, dass auch die ethisch-moralisch überlagerte Frage nach der Regulierung der Präimplantationsdiagnostik zumindest zu einem gewissen Grad von parteipolitischen Faktoren determiniert wurde, da die in den Reden - wo ein Abweichen von der ggf. bestehenden Parteilinie weniger Implikationen für den politischen wie legislativen Prozess beinhaltet als in den (namentlichen) Abstimmungen - eingenommenen Positionen nicht oder nur teilweise mit dem Abstimmungsverhalten der einzelnen Abgeordneten übereinstimmen. ${ }^{2}$

Um die Position der Abgeordneten zur Regulierung der PID zu bestimmen, ${ }^{3}$ werden die Redebeiträge aller Abgeordneten, die in einer der beiden Lesungen zur Regulierung der PID eine Rede gehalten oder zu Protokoll gegeben haben, einer quantitativen Inhaltsanalyse unterzogen. Wir greifen dabei auf das allein auf einem Vergleich der Worthäufigkeiten basierende, von Slapin und Proksch (2008) entwickelte Wordfish-Verfahren zurück (vgl. auch Proksch/Slapin 2009; Marcinkiewicz/Tepe 2012). Um die für Wordfish erforderlichen Ankerpunkte in Form der Formulierung

2 Vergleiche zu den Verzerrungen zwischen eigentlicher und in einer namentlichen Abstimmung tatsächlich eingenommenen Position etwa Carubba/Gabel/Hug (2008) und Hug (2010).

3 Von den im März 2011621 Mitgliedern des Deutschen Bundestags haben 91 eine Rede im Bundestag gehalten oder zu Protokoll gegeben. 
liberaler bzw. permissiver und konservativ-restriktiver Positionen in einer Rede setzen zu können, wurden die Reden der Abgeordneten systematisch und automatisiert mit Positionspapieren kirchlicher Akteure und ärztlichen bzw. wissenschaftlichen Verbänden verglichen. ${ }^{4}$ Durch die Anwendung des Wordfish-Verfahrens ist es möglich, für die Abgeordneten, die sich mit Redebeiträgen an der parlamentarischen Debatte beteiligt haben, die zur Frage der Regulierung der Präimplantationsdiagnostik zum Ausdruck gebrachte Position zu ermitteln. ${ }^{5}$

Die Informationen zur konfessionellen Zugehörigkeit der Abgeordneten, ihrem Geschlecht und der Frage, ob sie zum Zeitpunkt der Abstimmung im Juli 2011 Kinder hatten oder erwarteten, wurden den Kurzbiografien der Bundestagsmitglieder, wie sie auf der Homepage des Bundestages veröffentlicht sind, entnommen (vgl. auch Feldkamp 2010). ${ }^{6}$ Komplexer stellt sich die Ermittlung der Religiosität in den Wahlkreisen dar, für die ein Abgeordneter zuständig ist. Diese lässt sich auf Basis der offiziellen Statistik nicht ermitteln; die vom Bundeswahlleiter bereitgestellten Strukturdaten bieten leider keine Variable, die sich als Näherung für die Religiosität eines Wahlkreises einsetzen ließe. Angaben zur konfessionellen $\mathrm{Zu}-$ sammensetzung der Bevölkerung liegen zudem nur für ältere Zeiträume auf Landkreisebene vor und sind daher durch die mitunter deutlichen Unterschiede in den Zuschnitten von Land- und Wahlkreisen ebenfalls nicht als Indikator für die Religiosität eines Wahlkreises geeignet (vgl. Debus/Knill/Tosun 2012). Wir verwenden daher Daten der „German Longitudinal Election Study“ (Rattinger u.a. 2011) zur Bundestagswahl 2009, die nicht nur die Kirchgangshäufigkeit eines Befragten ermittelt, sondern auch eine Variable umfasst, die den Wahlkreis eines Befragten eindeutig identifiziert. Auf Basis dieser Informationen ist es mithilfe eines von Selb und Munzert (2011) entwickelten Imputationsverfahrens möglich, den Anteil der häufigen Kirchgänger selbst in den Wahlkreisen zu ermitteln, aus denen nur wenige oder gar keine Befragten in der Stichprobe zur deutschen Wahlstudie von 2009 vertreten sind. ${ }^{7}$ Die Abgeordneten werden dabei - unabhängig davon, ob sie ein

4 Die Ankerpunkte sind die Reden der Abgeordneten Christine Aschenberg-Dugnus (FDP), die wiederholt das Positionspapier des Wissenschaftsrates zitierte, sowie von Wolfgang Zöller (CSU), der im Plenum auf die Stellungnahme der Evangelischen Kirche in Deutschland zurückgriff.

5 Zur Ableitung der Wordfish-Positionen wurden die gängigen Vorbereitungsschritte zur Textskalierung sowie eine Entfernung sämtlicher Abgeordneten- und Parteinamen durchgeführt. Anhang 2 bietet weitere Übersichten zur Validierung der Skalierung.

6 Ob Abgeordnete ein Kind erwarteten, wurde darüber bestimmt, ob sie im Jahr 2012 Eltern waren oder nicht.

7 Wir möchten uns an dieser Stelle bei Simon Munzert und Peter Selb von der Universität Konstanz für die Unterstützung sowie die Bereitstellung der notwendigen Software bzw. R-Codes herzlich bedanken. Eine grafische Darstellung der für die Wahlkreise ermittelten Anteile von Kirchgängern mit und ohne Imputation findet sich in Anhang 1. 
Direkt- oder Listenmandat besitzen - jeweils den Wahlkreisen zugeordnet, in denen sie als Direktkandidat bei der Bundestagswahl 2009 angetreten waren. Für diejenigen Abgeordneten, die in keinem Wahlkreis kandidiert hatten, wurde der Standort ihres Abgeordnetenbüros ermittelt und der entsprechende Wahlkreis zugeordnet.

Bei unseren Analysen stellt somit jeder Bundestagsabgeordnete einen Fall dar. ${ }^{8}$ Die abhängigen Variablen geben erstens Auskunft darüber, welche Gesetzesinitiative ein Abgeordneter mit initiiert, zweitens welche Position er zur Regulierung der PID in seinen Parlamentsreden zum Ausdruck gebracht und drittens wie er in der namentlichen Abstimmung votiert hat. Der Übersichtlichkeit halber konzentrieren wir uns bei der Analyse der Initiierung und der Abstimmung auf die maßgeblich von Ulrike Flach (FDP) vertretene Vorlage, die die permissive Regulierung der PID vorsah. In diesen beiden Schritten der Untersuchung ist die abhängige Variable dichotom codiert, wobei der Wert ", “ diejenigen Abgeordneten kenntlich macht, die die permissive Gesetzesinitiative in der Vorbereitungszeit beziehungsweise in der namentlichen Abstimmung unterstützt haben. Daher kommen bei der Analyse logistische Regressionsmodelle zur Anwendung. Demgegenüber ergeben sich aus der inhaltsanalytischen Auswertung der Debattenbeiträge mit Wordfish Positionen der Abgeordneten auf einer Skala, so dass hier OLS-Regressionsmodelle geschätzt werden.

Im folgenden Abschnitt werden die Ergebnisse der Analyse in deskriptiver wie analytischer Form präsentiert.

\section{Ergebnisse}

Um zu überprüfen, inwiefern das legislative Handeln bei der Debatte und der Abstimmung über die Präimplantationsdiagnostik vom persönlichen Kontext der Bundestagsabgeordneten einerseits und den Einstellungen der Wähler im jeweils betreuten Wahlkreis andererseits beeinflusst war, analysieren wir zunächst die Bestimmungsfaktoren für die Unterstützung der Gesetzesinitiative, die eine permissive Regulierung der PID vorsieht. Im Anschluss daran beschreiben und untersuchen wir die in den Redebeiträgen der Abgeordneten eingenommenen Positionen zur Präimplantationsdiagnostik, um uns im dritten und letzten Analyseschritt den Deter-

8 Während bei der Analyse der Gesetzesinitiierung alle 621 Personen, die im März 2011 Mitglieder des Bundestags waren, die Grundgesamtheit in unserer Analyse darstellen, besteht bei der Analyse der Parlamentsreden die Grundgesamtheit nur aus den 91 Abgeordneten, die auch einen Redebeitrag geleistet haben. Um die Determinanten des Abstimmungsverhaltens zu ermitteln, ziehen wir als Basis nur die Bundestagsmitglieder heran, die mit „Ja“ oder „Nein“ gestimmt haben. Enthaltungen und Abwesenheit werden nicht berücksichtigt. 
Das legislative Verhalten von Bundestagsabgeordneten

minanten des Abstimmungsverhaltens zur legislativen Vorlage der permissiven Regelung zuzuwenden.

\section{a) Determinanten der Initiierung der permissiven Gesetzesvorlage zur Regu- lierung der PID}

Tabelle 2 gibt die Ergebnisse einer logistischen Regression wieder, deren abhängige Variable Informationen dazu umfasst, ob ein Mitglied des Bundestages die permissiv-liberale Gesetzesinitiative zur Regulierung der PID unterzeichnet hat oder nicht. Dies ermöglicht die empirische Überprüfung der Hypothesen 1 a, 2 a und 3 a und des in der vierten Hypothese erwarteten Zusammenhangs. Wir differenzieren dabei zwischen zwei Modellen. Während das erste Modell lediglich die aus den hier herangezogenen theoretischen Ansätzen ableitbaren erklärenden Variablen umfasst, bezieht Modell 2 zusätzlich die Fraktionszugehörigkeit mit in die Analyse ein. Damit kann überprüft werden, ob auch parteipolitische oder ideologische Faktoren eine zentrale Rolle spielten.

Beide Modelle unterstützen weitgehend unsere Erwartungen. So ist es umso unwahrscheinlicher, dass ein Abgeordneter die permissive Gesetzesvorlage einbringt bzw. unterzeichnet, je größer in dem Wahlkreis, den er repräsentiert, der Anteil der Bevölkerung mit einer hohen Kirchgangshäufigkeit ist. Darüber hinaus finden wir Evidenz dafür, dass Abgeordnete katholischen Glaubens eine signifikant geringere Chance aufweisen, den permissiven Vorschlag zu unterstützen. Für evangelische und konfessionslose Abgeordnete ergibt sich hingegen kein signifikanter Effekt. Hingegen weisen weibliche Bundestagsabgeordnete im Vergleich zu ihren Kollegen eine höhere Chance auf, den für eine liberal-permissive Regulierung der PID plädierenden Vorschlag zu unterstützen. Dies gilt insbesondere dann, wenn die weiblichen Mitglieder des Bundestages Kinder haben. Diese Zusammenhänge bleiben auch dann bestehen, wenn für die Fraktionszugehörigkeit kontrolliert wird (Modell 2). Von dieser geht - trotz des ethisch-moralischen Hintergrunds der Thematik ein nicht unbedeutender Effekt auf die legislative Initiativtätigkeit aus. So erhöht sich nicht nur deutlich die Erklärungskraft im Vergleich zum ersten Modell, sondern es zeigt sich auch, dass Abgeordnete der Union - im Vergleich zu Mitgliedern der SPD-Fraktion - eine signifikant geringere Chance aufweisen, den permissiven Vorschlag zu unterstützen, wohingegen Abgeordnete aus Reihen der Freien Demokraten mit einer signifikant höheren Wahrscheinlichkeit dazu neigen, den maßgeblich von ihrer Fraktionskollegin Ulrike Flach getragenen Vorschlag mit zu unterzeichnen. 
Markus Baumann, Marc Debus und Jochen Müller

Tabelle 2: Determinanten der Initiierung der permissiven Gesetzesvorlage zur Regulierung $\operatorname{der} P I D$

\begin{tabular}{lcc}
\hline & Modell 1 & Modell 2 \\
\hline Katholisch & $-1,34^{* *}$ & $-0,98^{* *}$ \\
& $(0,26)$ & $(0,32)$ \\
Evangelisch & $-0,05$ & 0,13 \\
& $(0,21)$ & $(0,26)$ \\
Konfessionslos & 0,17 & 0,43 \\
Männl. MdB mit Kind & $(0,45)$ & $(0,48)$ \\
Weibl. MdB ohne Kind & $-0,06$ & 0,18 \\
Weibl. MdB mit Kind & $(0,23)$ & $(0,27)$ \\
& 0,16 & $0,59^{+}$ \\
Kirchgangshäufigkeit im Wahlkreis & $(0,29)$ & $(0,32)$ \\
Bündnis 90/Die Grünen & $0,48^{+}$ & $0,73^{*}$ \\
CDU/CSU & $(0,27)$ & $(0,30)$ \\
Die Linke & $-3,22^{*}$ & $-5,27^{* *}$ \\
FDP & $(1,58)$ & $(1,84)$ \\
& & $-0,89^{* *}$ \\
Konstante & & $(0,34)$ \\
& & $-1,07^{* *}$ \\
N & & $(0,28)$ \\
Pseudo R & & $-0,63^{*}$ \\
\hline
\end{tabular}

Anmerkungen: Die abhängige Variable zeigt an, ob ein Abgeordneter die permissive Initiative unterstützt hat. Die Referenzkategorie bilden männliche Abgeordnete ohne Kinder, die keine Angaben zu ihrer Konfessionszugehörigkeit gemacht haben. Signifikanzniveaus: $+=p<0,1 *^{*}=p<0,05$; $* *=\mathrm{p}<0,01$.

Zur Interpretation der hohen Erklärungskraft der Fraktionsangehörigkeit muss in diesem Zusammenhang auch darauf hingewiesen werden, dass dies nicht zwingend auf eine parteipolitische Orientierung der Abgeordneten in dieser Sachfrage hindeutet. Vielmehr entfalten die im zweiten Modell in Tabelle 2 enthaltenen Variablen zur Fraktionszugehörigkeit schon allein deshalb einen gewissen Erklärungswert, weil sich in Parteien Abgeordnete mit ähnlichen Weltbildern zusammenfinden (Burden 2007: 39). Der Einschluss der Fraktionszugehörigkeit kann somit als eine Kontrollvariable betrachtet werden, die den Einfluss - durch die von uns betrachteten persönlichen Merkmale nicht erfasster - (ideologischer) Eigenschaften der Abgeordneten abbildet. Dass sich dennoch signifikante Effekte der Variablen er- 
geben, die die persönlichen Eigenschaften der Abgeordneten sowie der Bevölkerung in den ihnen zugeordneten Wahlkreisen widerspiegeln, unterstützt unsere Erwartungen. Dies gilt insbesondere für die Hypothesen 1 a, 2 a und 4: Sowohl die Religiosität im Wahlkreis als auch die Zugehörigkeit eines Abgeordneten zur katholischen Kirche spielen eine entscheidende Rolle für die Unterstützung der permissiven Gesetzesvorlage hinsichtlich der Regulierung der PID. Zudem spielt eine entscheidende Rolle, ob es sich um männliche oder weibliche Abgeordnete handelt, wobei Letztere insbesondere dann eine liberal-permissive Regulierung der PID unterstützen, wenn sie Kinder haben bzw. erwarteten.

\section{b) Determinanten der in den Reden vertretenen Positionen zur PID}

Im Vergleich zur Analyse der Unterstützung der Vorlage, die eine permissive Regulierung der PID vorsieht, ergibt sich keine so deutliche Evidenz für unsere in den Hypothesen 1 b, 2 b, 3 b und 4 formulierten Erwartungen, wenn die mit dem Wordfish-Verfahren auf der Basis von Parlamentsreden in der Debatte zur Präimplantationsdiagnostik ermittelte Position der Abgeordneten untersucht wird. Dies kann allerdings auch damit zusammenhängen, dass natürlich nicht alle, sondern nur 91 Mitglieder des Bundestages sich mit einem Redebeitrag an dieser Debatte beteiligten.

In Abbildung 2 a sind die Ausprägungen der ermittelten Positionen zur PID getrennt nach Fraktionszugehörigkeit mithilfe eines sogenannten „Boxplots“ wiedergegeben. ${ }^{9}$ Auf Basis der in den Reden eingenommenen Positionen zur Präimplantationsdiagnostik ergeben sich nur geringe Unterschiede zwischen den Fraktionen. Lediglich die Redner aus den Reihen der Liberalen vertraten mit nur einer Ausnahme Positionen für eine eher permissive Regulierung der PID. Auch die sozialdemokratischen Abgeordneten, die einen Redebeitrag lieferten, waren tendenziell wenn auch mit einer etwas größeren Spannweite - für eine begrenzte Anwendungsmöglichkeit der PID. Mitglieder der Unionsfraktion nahmen in ihren Reden im Durchschnitt eher skeptisch bis ablehnende Positionen ein - allerdings mit einigen Ausnahmen, wie die Reichweite des Balkens beim Eintrag von CDU/CSU in Abbildung 2 a zeigt. Während sich auch bei der Fraktion der Linken eine große Spannweite in den von ihren Vertretern eingenommenen Position zur PID ergibt, macht

9 Ein Boxplot liefert in grafischer Form Informationen über die Verteilung einer Variablen. Während die „Box“ den Bereich angibt, in dem die mittleren $50 \%$ der Daten liegen und der Strich innerhalb der Box den Wert des Median angibt, so weisen die strichartigen Verlängerungen der Box auf die Streuung der übrigen Werte der entsprechenden Variablen hin. Extreme Ausreißer sind durch separate Punkte gekennzeichnet. 
die Inhaltsanalyse der Äußerungen in der Bundestagsdebatte deutlich, dass innerhalb der Fraktion der Bündnisgrünen eine im Durchschnitt moderate und gleichzeitig relativ kohäsive Einstellung zur Regulierung der Präimplantationsdiagnostik vorherrschte.

Differenziert man die ermittelten Positionen zur PID nach dem Geschlecht der Abgeordneten, so zeigen sich nur geringe Unterschiede zwischen männlichen und weiblichen Bundestagsmitgliedern (vgl. Abbildung 2 b). Folgt man dem Wert des Median, der durch die Linie in der grau ausgefüllten Box markiert ist, so neigen die Rednerinnen in ihren Beiträgen zur PID-Debatte eher zu permissiveren Positionen als ihre männlichen Kollegen. Ein klarer Unterschied zeigt sich hingegen, wenn entlang der Konfessionen differenziert wird: Bis auf drei Ausnahmen nehmen katholische Abgeordnete in ihren Redebeiträgen deutlich konservativ-restriktivere Positionen zur Regulierung der Präimplantationsdiagnostik ein als Bundestagsmitglieder, die konfessionslos sind, einer anderen Religion angehören oder ihre Konfession nicht öffentlich angeben.

Abbildung 2: Verteilung der auf Grundlage der Parlamentsreden ermittelten Position gegenüber der PID (je höher der Wert, desto permissiv-liberaler die Position)

\section{(a) Fraktion}

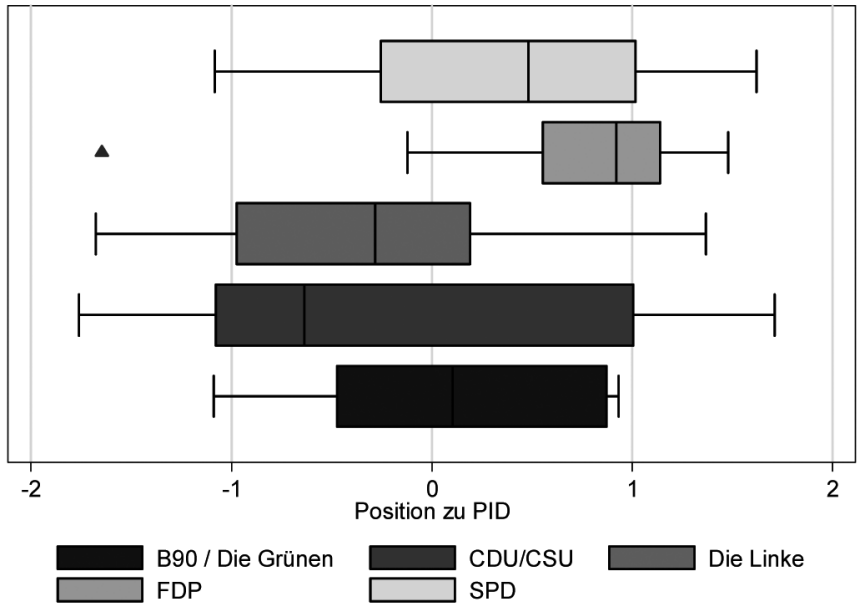




\section{(b) Geschlecht}

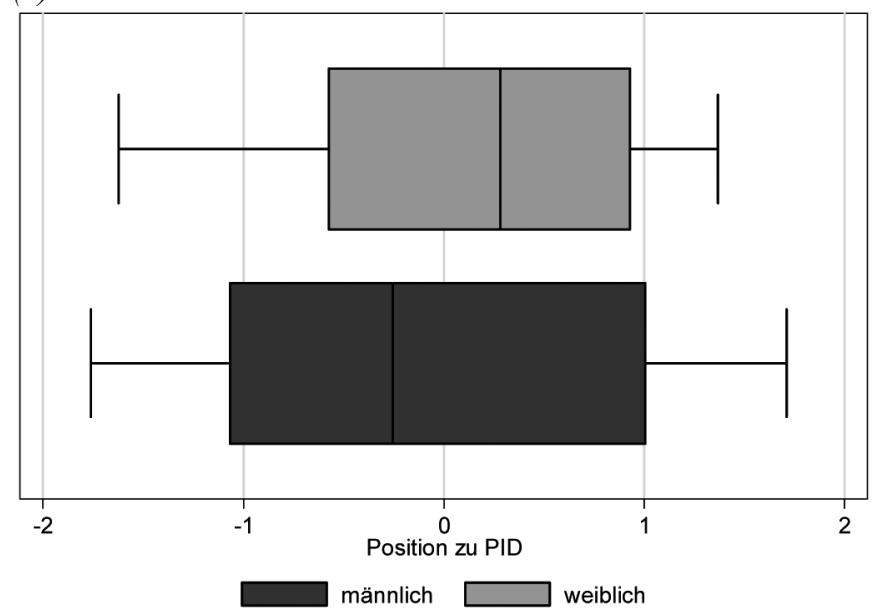

(c) Konfession

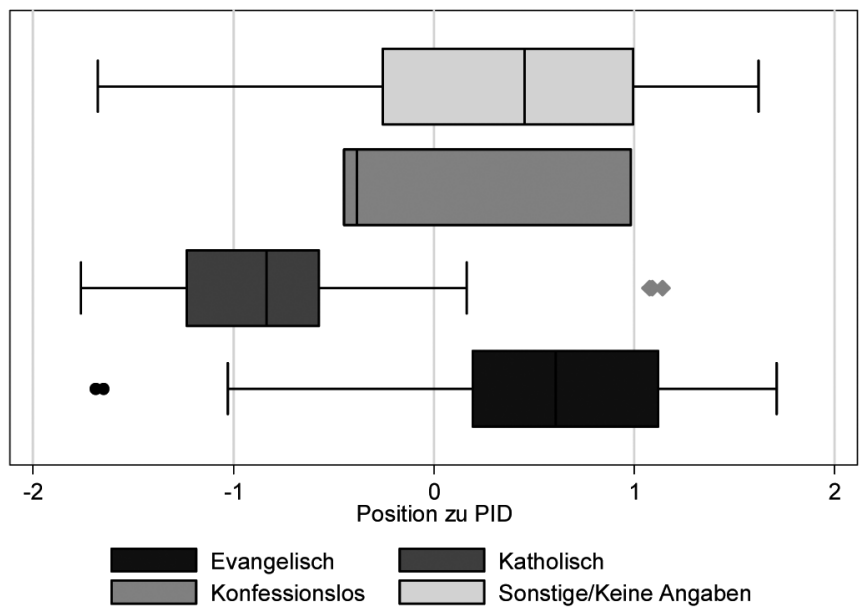

Verwendet man die auf Grundlage der Reden der 91 Abgeordneten ermittelten Positionen zur PID als abhängige Variable in einer linearen Regressionsanalyse und zieht wiederum die theoretisch hergeleiteten Faktoren als erklärende Variablen heran, so zeigt sich nur ein robuster Effekt der Zugehörigkeit eines MdB zur römischkatholischen Kirche: Abgeordnete katholischen Glaubens haben in ihren Beiträgen 
zur PID-Debatte eine restriktivere, konservativere Position eingenommen als die anderen, nicht-katholischen Redner. Die Effekte der Variablen, die Informationen zum Geschlecht eines Abgeordneten und zum Anteil der häufigen Kirchgänger in den Wahlkreisen umfassen, weisen zwar die erwartete Richtung auf, die auf den gängigen Signifikanzniveaus allerdings nicht verschieden von Null ist.

Tabelle 3: Determinanten der in den Debattenbeiträgen eingenommenen Position eines Abgeordneten zur permissiven Vorlage zur Regulierung der PID

\begin{tabular}{|c|c|c|}
\hline & Modell 1 & Modell 2 \\
\hline Katholisch & $\begin{array}{l}-0,93^{* *} \\
(0,23)\end{array}$ & $\begin{array}{l}-0,94^{* *} \\
(0,27)\end{array}$ \\
\hline Evangelisch & $\begin{array}{c}0,24 \\
(0,25)\end{array}$ & $\begin{array}{c}0,12 \\
(0,26)\end{array}$ \\
\hline Konfessionslos & $\begin{array}{l}-0,34 \\
(0,53)\end{array}$ & $\begin{array}{l}-0,19 \\
(0,53)\end{array}$ \\
\hline Männl. MdB mit Kind & $\begin{array}{c}0,27 \\
(0,27)\end{array}$ & $\begin{array}{c}0,13 \\
(0,27)\end{array}$ \\
\hline Weibl. MdB ohne Kind & $\begin{array}{c}0,39 \\
(0,32)\end{array}$ & $\begin{array}{c}0,38 \\
(0,31)\end{array}$ \\
\hline Weibl. MdB mit Kind & $\begin{array}{c}0,39 \\
(0,30)\end{array}$ & $\begin{array}{c}0,19 \\
(0,30)\end{array}$ \\
\hline Kirchgangshäufigkeit im Wahlkreis & $\begin{array}{l}-1,16 \\
(1,51)\end{array}$ & $\begin{array}{l}-2,39 \\
(1,57)\end{array}$ \\
\hline Bündnis 90/Die Grünen & & $\begin{array}{l}-0,10 \\
(0,33)\end{array}$ \\
\hline CDU/CSU & & $\begin{array}{l}-0,14 \\
(0,29)\end{array}$ \\
\hline Die Linke & & $\begin{array}{l}-0,77^{*} \\
(0,36)\end{array}$ \\
\hline FDP & & $\begin{array}{c}0,38 \\
(0,36)\end{array}$ \\
\hline Konstante & $\begin{array}{c}0,07 \\
(0,28) \\
\end{array}$ & $\begin{array}{c}0,42 \\
(0,34) \\
\end{array}$ \\
\hline $\mathrm{N}$ & 91 & 91 \\
\hline Korrigiertes $\mathrm{R}^{2}$ & 0,21 & 0,25 \\
\hline
\end{tabular}

Anmerkungen: Die abhängige Variable gibt die Position eines Abgeordneten an, die er in seinem Redebeitrag eingenommen hat. Höhere Werte implizieren permissiv-liberalere Haltungen gegenüber der PID. Die Referenzkategorie bilden männliche Abgeordnete ohne Kinder, die keine Angaben zu ihrer Konfessionszugehörigkeit gemacht haben. Signifikanzniveaus: $+=p<0,1 ; *=p<0,05 ; * *=p<0,01$. 
Das legislative Verhalten von Bundestagsabgeordneten

\section{c) Determinanten der Haltung der Abgeordneten in der namentlichen Ab- stimmung}

Die in Tabelle 4 ausgewiesenen Ergebnisse zeigen, ob und inwiefern das schlussendliche Abstimmungsergebnis und damit die letzte der drei im vorliegenden Beitrag untersuchten Stufen des legislativen Prozesses zur Regulierung der PID von den persönlichen Eigenschaften der Abgeordneten und den - anhand der Religiosität der Bevölkerung in den Wahlkreisen ermittelten - Präferenzen der Wählerschaft vor Ort beeinflusst wird. Somit werden die Hypothesen $1 \mathrm{c}, 2 \mathrm{c}$ und $3 \mathrm{c}$ sowie 4 einem empirischen Test unterzogen. Analog zu den Determinanten der Initiierung zeigt sich auch im Rahmen der Analyse des Abstimmungsverhaltens, dass sowohl die Zugehörigkeit zur römisch-katholischen Kirche als auch die Stärke der Religiosität in den Wahlkreisen einen Einfluss auf die Entscheidungsfindung der Abgeordneten hat: Ist ein Abgeordneter katholisch, dann lehnt er mit signifikant höherer Wahrscheinlichkeit den permissiven Vorschlag ab. Gleiches gilt bei einer Zunahme des Anteils kirchengebundener Wähler in den Wahlkreisen: Je höher dieser ist, desto eher lehnt ein Bundestagsmitglied - unter Kontrolle aller weiteren Faktoren, insbesondere auch der Fraktionszugehörigkeit (siehe Modell 2 in Tabelle 4) - die permissive Gesetzesvorlage zur Handhabung der Präimplantationsdiagnostik ab.

Ein ausschlaggebender Effekt kommt auch der Fraktionszugehörigkeit der Abgeordneten beim Abstimmungsverhalten zu. So geht - wie auch bei der Analyse der Initiierung der permissiven Vorlage - von allen Variablen, die die Parteizugehörigkeit kennzeichnen, ein statistisch signifikanter Effekt aus. Während im Vergleich zu den Mitgliedern der SPD-Fraktion die Angehörigen von Union, Bündnis 90/Die Grünen und der Linken mit einer höheren Chance den permissiven Vorschlag ablehnen, so steigt die Wahrscheinlichkeit, diesem Vorschlag zuzustimmen, in signifikanter Weise an, wenn ein Abgeordneter Mitglied der FDP-Fraktion ist. Hingegen sind keine Effekte der Variablen mehr nachweisbar, die sich auf die persönlichen Eigenschaften und den familiären Kontext der Bundestagsmitglieder beziehen. Offenbar spielt bei parlamentarischen Abstimmungen - selbst bei solchen, die ohne „Fraktionszwang“" stattfinden - die Zugehörigkeit zu einer Partei und deren parlamentarischer Gruppe eine wichtigere Rolle als bei den vorgelagerten Stufen des legislativen Prozesses wie der Initiierung und Diskussion von Gesetzesvorschlägen, so dass persönliche Hintergründe und Erfahrungen in dieser Stufe des legislativen Prozesses weniger zum Tragen kommen als in der Phase der Initiierung von Politikvorschlägen. Ein Grund hierfür mag sein, dass die Partei der dominante „Prinzipal" im Rahmen der schlussendlichen Handlungen eines Abgeordneten selbst bei „freigegebenen“ Abstimmungen ist, so dass im Hinblick auf die Perzeption der in- 
Tabelle 4: Determinanten des Abstimmungsverhaltens über den liberal-permissiven Vorschlag

\begin{tabular}{|c|c|c|}
\hline & Modell 1 & Modell 2 \\
\hline Katholisch & $\begin{array}{l}-1,46^{* *} \\
(0,23)\end{array}$ & $\begin{array}{l}-1,07^{* *} \\
(0,30)\end{array}$ \\
\hline Evangelisch & $\begin{array}{c}0,01 \\
(0,22)\end{array}$ & $\begin{array}{c}0,27 \\
(0,29)\end{array}$ \\
\hline Konfessionslos & $\begin{array}{c}0,27 \\
(0,55)\end{array}$ & $\begin{array}{c}0,49 \\
(0,58)\end{array}$ \\
\hline Männl. MdB mit Kind & $\begin{array}{l}-0,18 \\
(0,23)\end{array}$ & $\begin{array}{c}0,00 \\
(0,27)\end{array}$ \\
\hline Weibl. MdB ohne Kind & $\begin{array}{l}-0,25 \\
(0,30)\end{array}$ & $\begin{array}{l}-0,00 \\
(0,33)\end{array}$ \\
\hline Weibl. MdB mit Kind & $\begin{array}{l}-0,07 \\
(0,28)\end{array}$ & $\begin{array}{l}-0,03 \\
(0,32)\end{array}$ \\
\hline Kirchgangshäufigkeit im Wahlkreis & $\begin{array}{l}-4,79^{* *} \\
(1,52)\end{array}$ & $\begin{array}{l}-6,14^{* *} \\
(1,76)\end{array}$ \\
\hline Bündnis 90/Die Grünen & & $\begin{array}{l}-1,36^{* *} \\
(0,34)\end{array}$ \\
\hline $\mathrm{CDU} / \mathrm{CSU}$ & & $\begin{array}{l}-1,57^{* *} \\
(0,28)\end{array}$ \\
\hline Die Linke & & $\begin{array}{l}-1,03^{* *} \\
(0,35)\end{array}$ \\
\hline FDP & & $\begin{array}{c}1,99^{* *} \\
(0,51)\end{array}$ \\
\hline Konstante & $\begin{array}{l}1,44^{* *} \\
(0,24)\end{array}$ & $\begin{array}{c}1,66^{* *} \\
(0,33)\end{array}$ \\
\hline $\mathrm{N}$ & 586 & 586 \\
\hline Pseudo $\mathrm{R}^{2}$ & 0,10 & 0,25 \\
\hline
\end{tabular}

Anmerkung: Die abhängige Variable gibt an, ob ein Abgeordneter sich für die liberal-permissive Initiative zur Regulierung der PID in der Abstimmung entschieden hat. Die Referenzkategorie bilden männliche Abgeordnete ohne Kinder, die keine Angaben zu ihrer Konfessionszugehörigkeit gemacht haben. Signifikanzniveaus: $+=p<0,1 ; *=p<0,05 ; * *=p<0,01$.

haltlichen Standpunkte einer Partei durch die Wählerschaft das einheitliche Auftreten einer Partei nach außen verstärkt wird, was die Erfüllung des Ziels der Stimmenmaximierung erleichtern sollte. Zur Überprüfung dieser Interpretation ist jedoch noch weitergehende Forschungsarbeit nötig.

Zusammengefasst lässt sich festhalten, dass die Abgeordneten des Deutschen Bundestages bei ethisch-moralischen Themen nicht nur der Parteiideologie und -programmatik, sondern auch zu einem gewissen Grad ihren Erfahrungen und Werten, die sich aus dem persönlichen Kontext ergeben, sowie den Präferenzen ihrer lokalen Wählerschaft folgen. Zum einen stützt der Einfluss der persönlichen Charakteristika eines Abgeordneten auf sein legislatives Verhalten bei Thematiken mit 
ethisch-moralischem Hintergrund wie der PID zumindest in der Phase der Initiierung einer Gesetzesvorlage die theoretischen Überlegungen von Burden (2007). Zum anderen zeigt der Einfluss der Wahlkreiseigenschaften, der auch beim schlussendlichen Abstimmungsverhalten vorliegt, Evidenz für ein responsives Verhalten der Abgeordneten, das sich an den Präferenzen der lokalen Wähler (und auch der parteipolitischen Entscheidungsträger) orientiert. Dieses Ergebnis, das einen Einklang zwischen den Präferenzen des Elektorats und seinen Vertretern im Parlament belegt, zeigt, dass Abgeordnete offenbar auch bei religiös und ethisch-moralisch unterlegten Thematiken ihr legislatives Handeln und Entscheiden der Maxime der Nutzenmaximierung unterstellen: Wenn parlamentarische Repräsentanten den Interessen und Einstellungen der Mehrheit der (lokalen) Wähler und Parteimitglieder folgen, dann sollte dies die Chance erhöhen, bei kommenden Wahlen erneut als Kandidat nominiert und schließlich auch wiedergewählt zu werden.

\section{Schlussbetrachtung}

Ziel des vorliegenden Beitrags war die Analyse des Handelns und Entscheidens von Bundestagsabgeordneten bei religiös und ethisch-moralisch überlagerten Themen. Wir haben zum einen argumentiert, dass gewählte Repräsentanten - gemäß den gängigen Theorien des Verhaltens parteipolitischer Akteure - die Interessen der lokalen Wählerschaft in ihrer legislativen Aktivität berücksichtigen sollten, um ein besseres Wahlergebnis zu erzielen. Zum anderen haben wir - aufbauend auf Studien zur gesetzgeberischen Aktivität im amerikanischen Kongress - argumentiert, dass sich Abgeordnete insbesondere bei Debatten zu Themen, bei denen die Fraktionsdisziplin aufgrund der Thematik der Debatte ,aufgehoben“ ist, verstärkt von ihren persönlichen Erfahrungen und ihrem privaten Kontext leiten lassen, wenn es darum geht, zu legislativen Vorlagen ihre Meinung kundzutun.

Unsere Ergebnisse haben größtenteils die Erwartungen bestätigt: Sowohl die Interessenlage in den Wahlkreisen als auch persönliche Charakteristika der Abgeordneten wie deren Konfession, Geschlecht und Elternschaft spielen - zumindest bei der Auseinandersetzung um die Präimplantationsdiagnostik im Deutschen Bundestag - eine bedeutende Rolle. Diese Ergebnisse bieten Anreize für weitere Studien zu den Determinanten des Verhaltens von Parlamentariern in legislativen Entscheidungsprozessen. So sollte aus theoretischem Blickwinkel die gängige, rein auf Maximierung von Stimmen, Ämtern und Politikinhalten ausgerichtete Perspektive zur Erklärung der Muster von Handeln und Entscheiden politischer Akteure um die persönlichen Erfahrungen und privaten Kontextfaktoren, die die einzelnen Entscheidungsträger prägen, auch für parlamentarische Systeme mit hoher Parteidis- 
ziplin erweitert werden, um so die politische Wirklichkeit besser abbilden zu können. Zudem bietet sich ein Vergleich der Ergebnisse mit den Determinanten des legislativen Verhaltens bei Themengebieten an, die von den „gängigen“ Konfliktlinien, die Parteienwettbewerb und politischen Prozess eines Landes prägen, weitgehend abgedeckt werden. Der persönliche Hintergrund eines Abgeordneten sollte hier weniger oder gar nicht relevant für sein Handeln und Entscheiden sein. Auch bietet sich die Einnahme einer vergleichenden Perspektive an, um die Effekte unterschiedlicher Wahl- und Parteiensysteme auf die Responsivität - im Sinne eines Zusammenhangs zwischen der Interessenlage der Wählerschaft und dem Handeln der Abgeordneten - zu untersuchen.

\section{Literatur}

Andeweg, Rudy B./Thomassen, Jacques, 2011: Pathways to Party Unity: Sanctions, Loyalty, Homogeneity, and Division of Labor in the Dutch Parliament, in: Party Politics 17 (5), 655-672.

Bergman, Torbjörn/Müller, Wolfgang C./Strøm, Kaare, 2000: Introduction: Parliamentary democracy and the chain of delegation, in: European Journal of Political Research 37 (3), 255-260.

Bowler, Shaun, 2010: Private members' bills in the UK parliament. Is there an 'electoral connection'?, in: Journal of Legislative Studies 16 (4), 476-494.

Bräuninger, Thomas/Debus, Marc, 2012: Parteienwettbewerb in den deutschen Bundesländern, Wiesbaden.

Bräuninger, Thomas/Brunner, Martin/Däubler, Thomas, 2012: Personal VoteSeeking in Flexible List Systems: How Electoral Incentives Shape Belgian MPs' Bill Initiation Behaviour, in: European Journal of Political Research 51 (5), 607-645.

Brunner, Martin, 2013: Parliaments and Legislative Activity: Motivations for Bill Introduction, Wiesbaden.

Burden, Barry C., 2007: Personal Roots of Representation, Princeton.

Carey, John C., 2009: Legislative Voting and Accountability, Cambridge.

Carubba, Clifford J./Gabel, Matthew/Hug, Simon, 2008: Legislative voting behavior, seen and unseen: A theory of roll-call vote selection, in: Legislative Studies Quarterly 33 (4), 543-572.

Debus, Marc, 2012: Sozialstrukturelle und einstellungsbasierte Determinanten des Wahlverhaltens und ihr Einfluss bei den Bundestagswahlen im Zeitverlauf: Westdeutschland 1976-2009, in: Rüdiger Schmitt-Beck (Hrsg.), Wählen in 
Deutschland. Sonderheft 45 der Politischen Vierteljahresschrift, Baden-Baden, 40-62.

Debus, Marc/Knill, Christoph/Tosun, Jale, 2012: Drum zahle, wer sich ewig bindet: Eine Analyse der Gebührenhöhe für eingetragene Lebenspartnerschaften in Baden-Württemberg, in: Politische Vierteljahresschrift 53 (1), 1-28.

Depauw, Sam/Martin, Shane, 2009: Legislative Party Discipline and Cohesion in Comparative Perspective, in: Kenneth Benoit/Daniela Giannetti (Hrsg.), IntraParty Politics and Coalition Governments, London, 103-120.

Dodson, Debra L., 2006: The Impact of Women in Congress, Oxford.

Engeli, Isabelle/Green-Pedersen, Christoffer/Larsen, Lars Thorup (Hrsg.), 2012, Morality Politics in Western Europe: Parties, Agendas and Policy Choices, Basingstoke.

Esaiasson, Peter/Heidar, Knut, 2000: Beyond Westminster and Congress - The Nordic Experience, Columbus.

Esser, Hartmut, 1999: Soziologie: Spezielle Grundlagen. Band 1: Situationslogik und Handeln, Frankfurt a. M.

Esser, Hartmut, 2001: Soziologie: Spezielle Grundlagen. Band 6: Sinn und Kultur, Frankfurt a. M.

Falter, Jürgen W./Schoen, Harald (Hrsg.), 2005: Handbuch Wahlforschung, Wiesbaden.

Feldkamp, Michael F., 2010: Deutscher Bundestag 1990 bis 2009: Parlaments- und Wahlstatistik für die 12. bis 17. Wahlperiode, in: Zeitschrift für Parlamentsfragen 41 (1), 3-17.

Gschwend, Thomas/Zittel, Thomas, 2007: Individualisierte Wahlkämpfe im Wahlkreis. Eine Analyse am Beispiel des Bundestagswahlkampfes von 2005, in: Politische Vierteljahresschrift 48 (2), 293-321.

Hartmann, Jürgen, 2007: Persönlichkeit und Politik, Wiesbaden.

Hug, Simon, 2010: Selection Effects in Roll Call Votes, in: British Journal of Political Science 40 (1), 225-235.

Ismayr, Wolfgang, 2000: Der Deutsche Bundestag, Opladen.

Kam, Christopher, 2009: Party Discipline and Parliamentary Government, Cambridge.

Kingdon, John W., 1989: Congressmen's Voting Decisions, Michigan.

Lipset, Seymour Martin/Rokkan, Stein, 1967: Cleavage Structures, Party Systems and Voter Alignments: An Introduction, in: Seymour Martin Lipset/Stein Rokkan (Hrsg.), Party Systems and Voter Alignments: Cross-National Perspectives, New York/London, 1-64. 
Manow, Philip, 2007: Electoral Rules and Legislative Turnover: Evidence from Germany's Mixed Electoral System, in: West European Politics 30 (1), 195-207. Manow, Philip, 2012: Wahlkreis- oder Listenabgeordneter, Typus oder Episode? Eine Sequenzanalyse der Wege in den Bundestag, in: Politische Vierteljahresschrift 53 (1), 53-78.

Marcinkiewicz Kamil/Tepe, Markus, 2012: Politische Positionen in Abgeordnetenbiographien. Eine quantitative Textanalyse der Kurzbiographien aus Kürschners Volkshandbuch für den 8. und 17. Deutschen Bundestag, in: Zeitschrift für Politikberatung 5 (2), 51-63.

Mead, George H., 1934: Mind, Self and Society: From the standpoint of a social behaviourist, Chicago.

Meidert, Nadine/Nebel, Kerstin, 2013: Moralpolitik am Beispiel von Einstellungen zum Schwangerschaftsabbruch in Deutschland. Eine vergleichende Längsschnittstudie von Gesellschaft und politischen Akteuren, in: Zeitschrift für Politikwissenschaft 23 (1), 77-102.

Müller, Wolfgang C., 2000: Political parties in parliamentary democracies: Making delegation and accountability work, in: European Journal of Political Research 37 (3), 309-333.

Pappi, Franz U./Brandenburg, Jens, 2010: Sozialstrukturelle Interessenlagen und Parteipräferenz in Deutschland: Stabilität und Wandel seit 1980, in: Kölner Zeitschrift für Soziologie und Sozialpsychologie 62 (4), 459-483.

Pappi, Franz U./Shikano, Susumu, 2002: Die politisierte Sozialstruktur als mittelfristig stabile Basis einer deutschen Normalwahl, in: Kölner Zeitschrift für Soziologie und Sozialpsychologie 54 (3), 444-475.

Pappi, Franz U./Shikano, Susumu, 2004: Ideologische Signale in den Wahlprogrammen der deutschen Bundestagsparteien 1980 bis 2002, MZES Working Papers 76.

Pappi, Franz U./Shikano, Susumu, 2007: Wahl- und Wählerforschung, Baden-Baden.

Patzelt, Werner J., 1989: Wahlkreisstil und Abgeordnetenrollen, in: Zeitschrift für Parlamentsfragen 20 (1), 114-150.

Patzelt, Werner, 2007: The Constituency Roles of MPs at the Federal and Länder Levels in Germany, in: Regional and Federal Studies 17 (1), 47-70.

Powell, Bingham G., 2004: The chain of responsiveness, in: Journal of Democracy 15 (1), 91-105.

Preidel, Caroline, 2011: Die soziale Logik der Moralpolitik: Determinanten des Abstimmungsverhaltens bei moralpolitischen Gesetzesinitiativen im Deutschen Bundestag, Masterarbeit, Universität Konstanz. 
Preidel, Caroline, 2012: Die Debatte zur Stammzellforschungspolitik im Deutschen Bundestag: Der Einfluss der Religion auf legislative Gewissensentscheidungen, unveröffentlichtes Arbeitspapier, Universität Konstanz.

Proksch, Sven-Oliver/Slapin, Jonathan B., 2009: How to Avoid Pitfalls in Statistical Analysis of Political Texts: The Case of Germany, in: German Politics 18 (3), 323-344.

Proksch, Sven-Oliver/Slapin, Jonathan B., 2012: Institutional Foundations of Legislative Speech, in: American Journal of Political Science 56 (3), 520-537.

Rattinger, Hans/Roßteutscher, Sigrid/Schmitt-Beck, Rüdiger/Weßels, Bernhard u. a., 2011: Zwischen Langeweile und Extremen: Die Bundestagswahl 2009, Baden-Baden.

Rebenstorf, Hilke/Weßels, Bernhard, 1989: Wie wünschen sich die Wähler ihre Abgeordneten? Ergebnisse einer repräsentativen Bevölkerungsfrage zum Problem der sozialen Repräsentativität des Deutschen Bundestags, in: Zeitschrift für Parlamentsfragen 20 (3), 408-424.

Rocca, Michael S./Sanchez, Gabriel, 2008: The Effect of Race and Ethnicity on Bill Sponsorship and Cosponsorship in Congress, in: American Politics Research 36 (1), 130-152.

Saalfeld, Thomas, 1995: Parteisoldaten und Rebellen: Eine Untersuchung zur Geschlossenheit der Fraktionen im Deutschen Bundestag (1949-1990), Opladen.

Saalfeld, Thomas, 2003: The Bundestag: Institutional Incrementalism and Behavioural Reticence, in: Kenneth Dyson/Klaus H. Goetz (Hrsg.), Germany, Europe, and the Politics of Constraint, Oxford, 73-96.

Schoen, Harald, 2012: Persönlichkeit, politische Präferenzen und politische Partizipation, in: Aus Politik und Zeitgeschichte 62 (49-50), 47-52.

Searing, Donald D., 1991: Roles, Rules, and Rationality in the New Institutionalism, in: American Political Science Review 85 (4), 1239-1260.

Selb, Peter/Munzert, Simon, 2011: Estimating Constituency Preferences from Sparse Survey Data Using Auxiliary Geographic Information, in: Political Analysis 19 (4), 455-470.

Sieberer, Ulrich, 2006: Party Unity in Parliamentary Democracies. A Comparative Analysis, in: Journal of Legislative Studies 12 (2), 150-178.

Sieberer, Ulrich, 2010: Behavioral Consequences of Mixed Electoral Systems. Deviating Voting Behavior of District and List MPs in the German Bundestag, in: Electoral Studies 29 (3), 484-496.

Sieberer, Ulrich, 2013: Using MP statements to explain voting behaviour in the German Bundestag: An individual level test of the Competing Principals Theory, in: Party Politics (online first). 
Simon, Herbert A., 1985: Human nature in politics: The dialogue of psychology with political science, in: The American Political Science Review 79 (2), 293-304.

Simon, Herbert A., 1995: Rationality in political behavior, in: Political Psychology $16(1), 45-61$.

Slapin, Jonathan B./Proksch, Sven-Oliver, 2008: A Scaling Model for Estimating Time-Series Policy Positions from Texts, in: American Journal of Political Science 52 (3), 705-722.

Stecker, Christian, 2011 a: Namentliche Abstimmungen als Währung individueller Verantwortlichkeit? Eine vergleichende Analyse der deutschen Länderparlamente, in: Zeitschrift für Vergleichende Politikwissenschaft 5 (2), 303-328.

Stecker, Christian, 2011 b: Bedingungsfaktoren der Fraktionsgeschlossenheit. Eine vergleichende Analyse der deutschen Länderparlamente, in: Politische Vierteljahresschrift 52 (3), 424-447.

Strøm, Kaare/Müller, Wolfgang C., 1999: Political Parties and Hard Choices, in: Wolfgang C. Müller/Kaare Strøm (Hrsg.), Policy, Office, or Votes? Cambridge, $1-35$.

Thurner, Paul W., 2000: The Empirical Application of the Spatial Theory of Voting in Multiparty Systems with Random Utility Models, in: Electoral Studies 19 (4), 493-517.

Wängnerud, Lena, 2009: Women in Parliaments: Descriptive and Substantive Representation, in: Annual Review of Political Science 12, 51-69.

Weßels, Bernhard, 2000: Gruppenbindung und Wahlverhalten: 50 Jahre Wahlen in der Bundesrepublik, in: Markus Klein/Wolfgang Jagodzinski/Ekkehard Mochmann/Dieter Ohr (Hrsg.), 50 Jahre Empirische Wahlforschung in Deutschland, Opladen, 129-155.

Korrespondenzanschrift:

Markus Baumann, M.A.

Mannheimer Zentrum für Europäische Sozialforschung (MZES)

Universität Mannheim

68131 Mannheim

E-Mail:mbaumann@uni-mannheim.de 
Prof. Dr. Marc Debus

Professur für Politische Wissenschaft III, Vergleichende Regierungslehre Fakultät für Sozialwissenschaften

Universität Mannheim

68131 Mannheim

E-Mail: marc.debus@uni-mannheim.de

Dr. Jochen Müller

Department of Government

University of Essex

Wivenhoe Park

Colchester, Essex

CO4 3SQ

E-Mail: jmueller@essex.ac.uk 


\section{Anhang 1: Kirchgangshäufigkeit in den Wahlkreisen}

Abbildung A1-1: Anteil der Befragten mit hoher Kirchgangshäufigkeit in den Wahlkreisen basierend auf den Daten der GLES

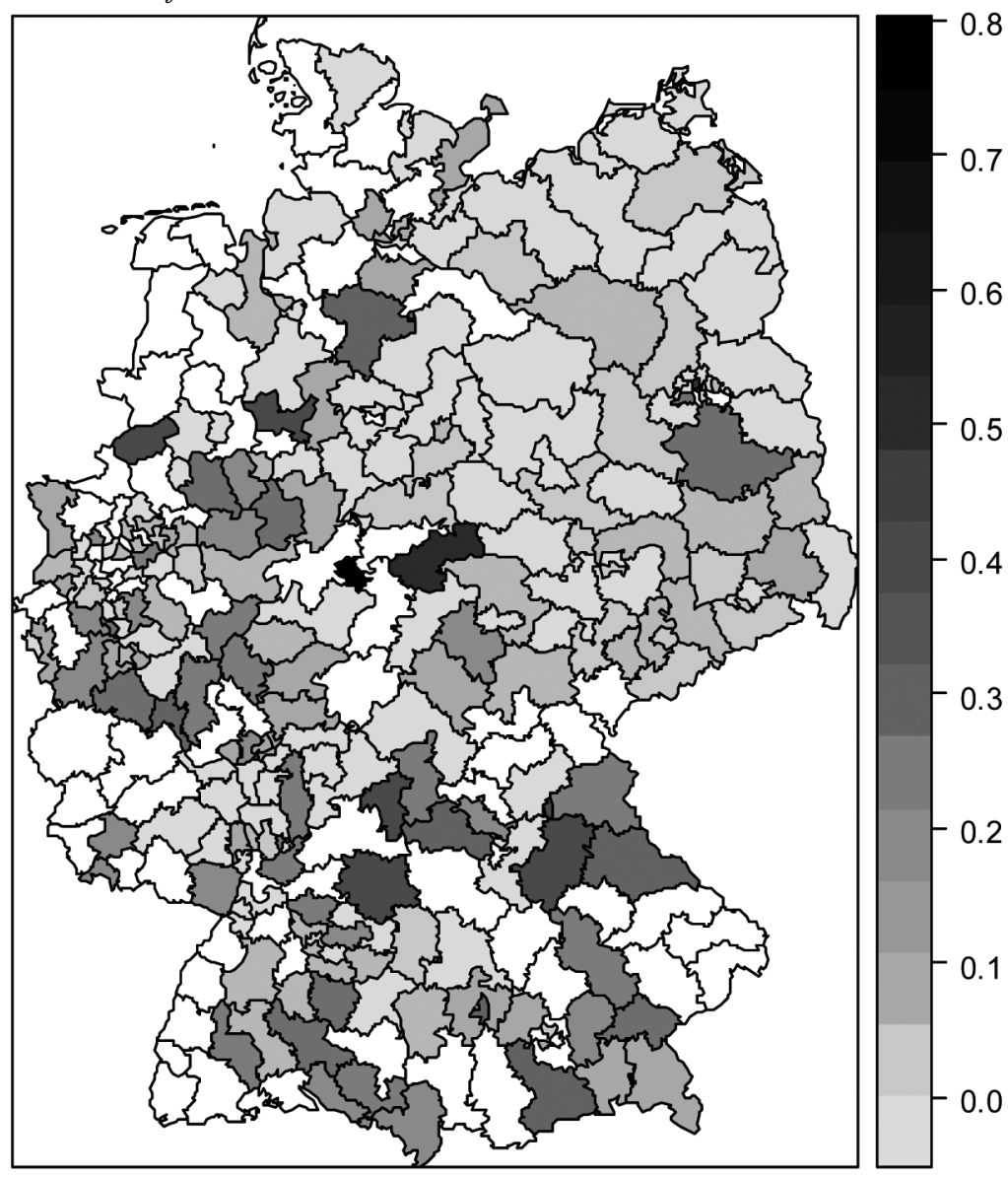

Anmerkung: Als hohe Kirchgangshäufigkeit gilt ein monatlicher oder häufigerer Besuch der Kirche. Dunklere Farben symbolisieren einen größeren Anteil von Kirchgängern; weißgefüllte Wahlkreise sind solche, die von den Befragten der GLES nicht abgedeckt werden. 


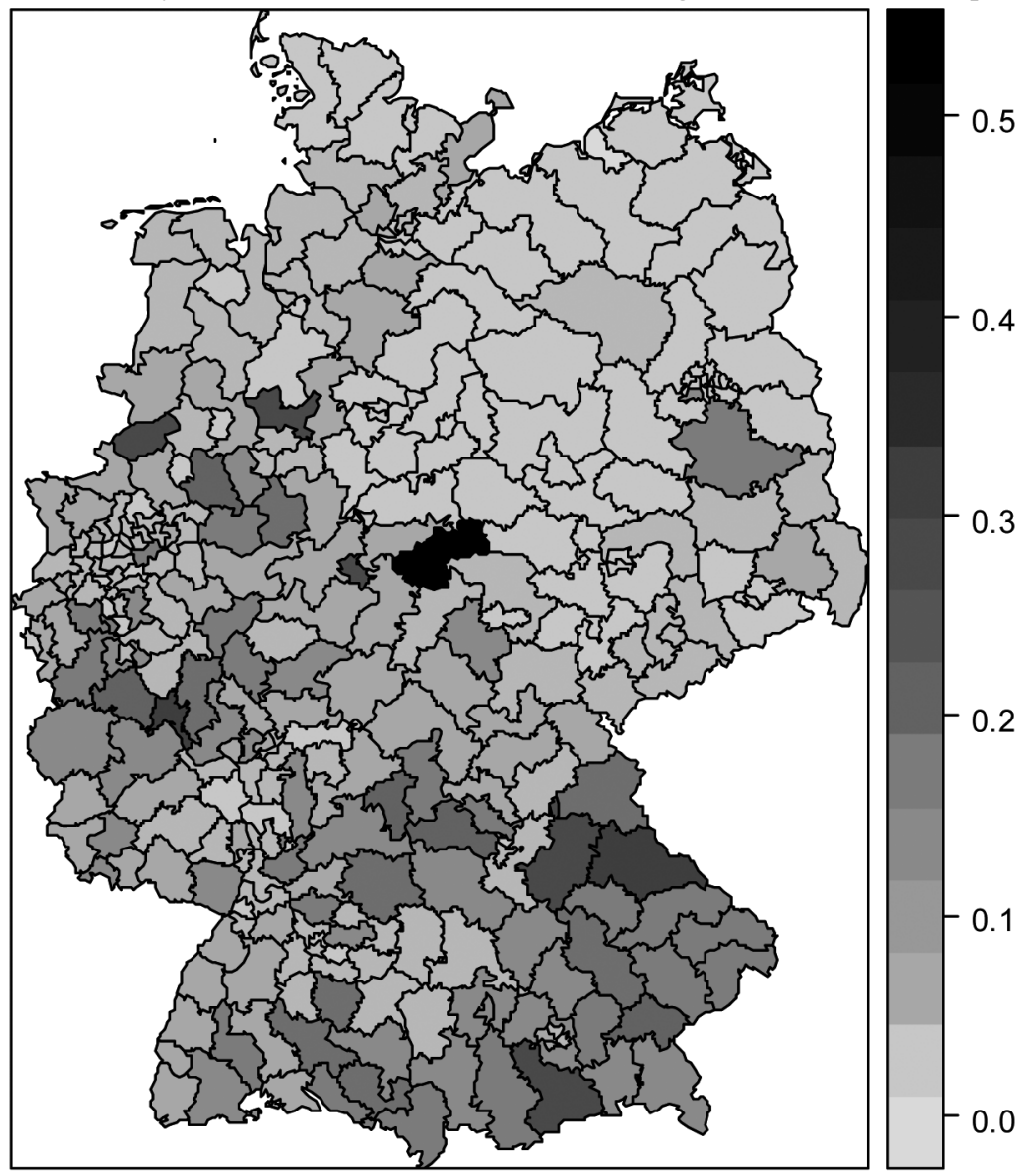

Anmerkung: Für Wahlkreise mit fehlenden Werten wird aufbauend auf ihrer geografischen Lage und dem in ihren jeweiligen Nachbarwahlkreisen vorhandenen Anteil von Kirchgängern ein entsprechender Wert imputiert. Wahlkreise mit vorhandenen Werten, die aber auf einer geringen Zahl von Befragten basieren, werden ebenfalls an die sie umgebenden Wahlkreise angenähert. 


\section{Anhang 2: Validierung der in den Reden bezogenen Positionen}

Abbildung A2-1: Verteilung der Positionen zur PID nach späterem Abstimmungsverhalten (je höher der Wert, desto permissiv-liberaler die Position)

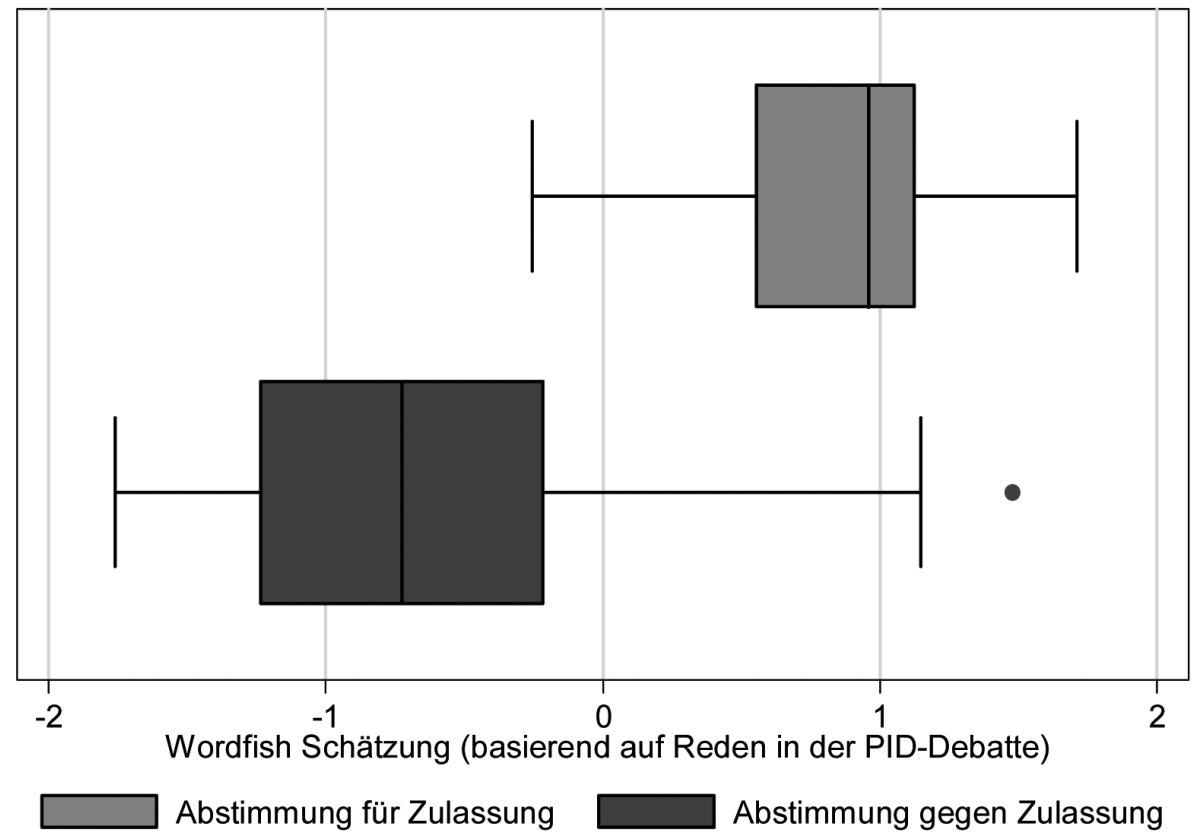


Das legislative Verhalten von Bundestagsabgeordneten

Abbildung A2-2: Wordfish-Positionen in den Reden der 24 Abgeordneten, die sowohl in der ersten als auch in der zweiten Lesung geredet haben

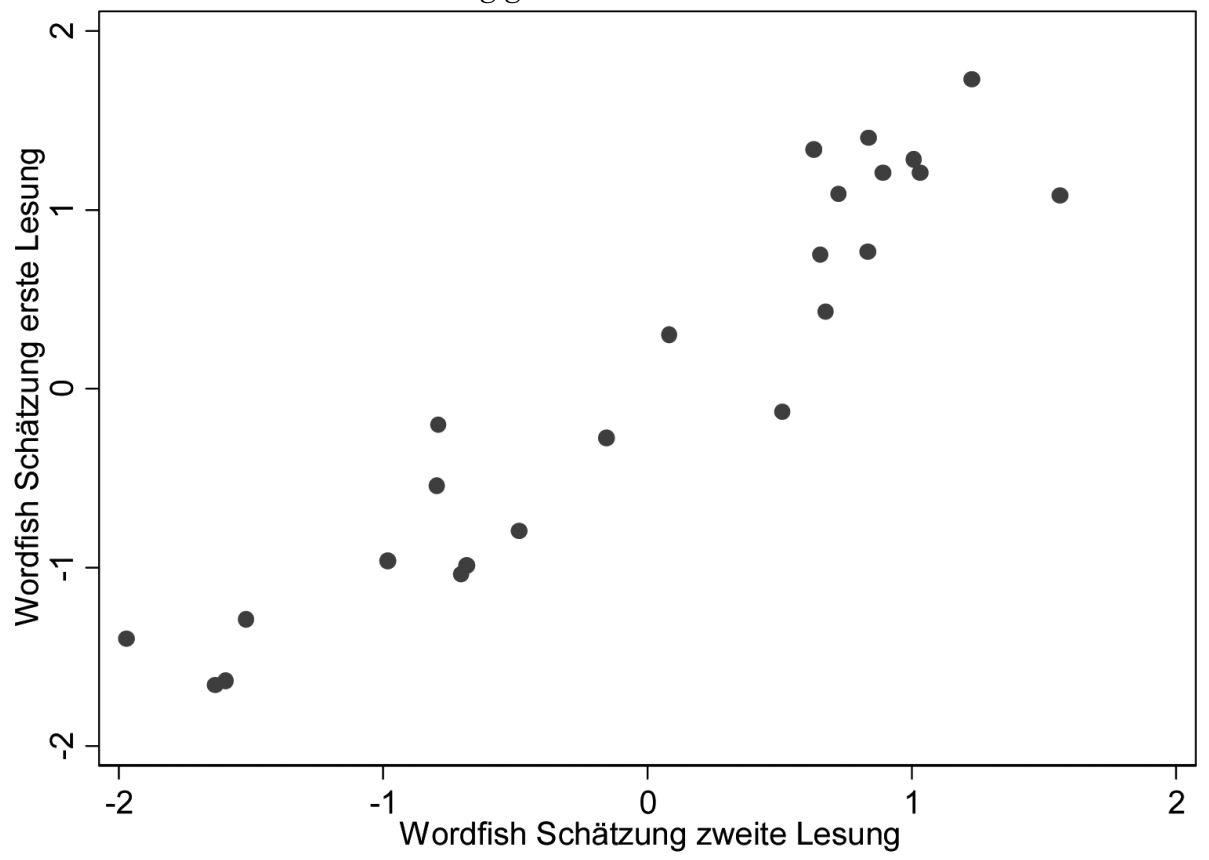

